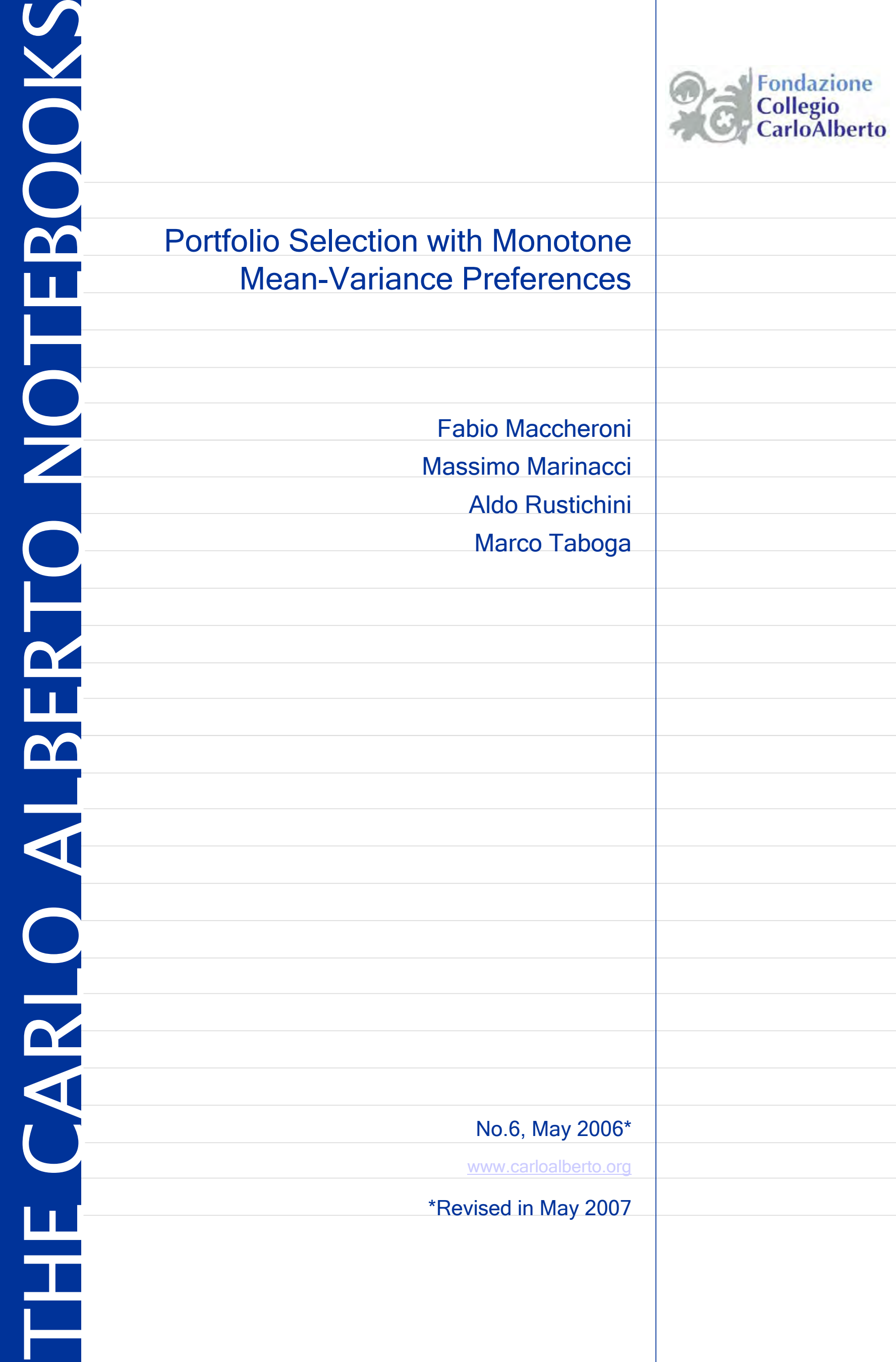




\title{
Portfolio Selection with Monotone Mean-Variance Preferences ${ }^{1}$
}

\author{
Fabio Maccheroni \\ Istituto di Metodi Quantitativi and IGIER, Università Bocconi \\ Massimo Marinacci \\ Collegio Carlo Alberto and Università di Torino \\ Aldo Rustichini \\ Department of Economics, University of Minnesota \\ Marco Taboga \\ Research Department, Banca d'Italia \\ Revision of January 2007
}

\begin{abstract}
${ }^{1}$ First circulated draft: December 2004. We thank Erio Castagnoli, Rose-Anne Dana, Paolo Guasoni, Jianjun Miao, Giovanna Nicodano, Francesco Sangiorgi, Giacomo Scandolo, Elena Vigna, and, especially, Dilip Madan, an associate editor, and two anonymous referees for helpful suggestions. We are also grateful for stimulating discussions to the participants at the VI Quantitative Finance Workshop (Milan, January 2005), SMAI 2005 (Evian, May 2005), RUD 2005 (Heidelberg, June 2005) and to the seminar audiences at Banca d'Italia, Boston University, Collegio Carlo Alberto, Università Bocconi, Università di Bologna, Università del Piemonte Orientale, Università di Salerno, and Università di Udine. Part of this research was done while the first two authors were visiting the Department of Economics of Boston University, which they thank for its hospitality. The authors also gratefully acknowledge the financial support of the Collegio Carlo Alberto, National Science Foundation (grant SES-0452477), and Università Bocconi.

(C) 2007 by Fabio Maccheroni, Massimo Marinacci, Aldo Rustichini, and Marco
\end{abstract}




\begin{abstract}
We propose a portfolio selection model based on a class of monotone preferences that coincide with mean-variance preferences on their domain of monotonicity, but differ where mean-variance preferences fail to be monotone and are therefore not economically meaningful. The functional associated to this new class of preferences is the best approximation of the mean-variance functional among those which are monotonic.

We solve the portfolio selection problem and we derive a monotone version of the CAPM, which has two main features: (i) it is, unlike the standard CAPM model, arbitrage free, (ii) it has empirically testable CAPM-like relations. The monotone CAPM has thus a sounder theoretical foundation than the standard CAPM and a comparable empirical tractability.
\end{abstract}

JEL classification: G11, G12

Keywords: Mean-Variance Preferences, CAPM

Taboga. Any opinions expressed here are those of the authors and not those of either the Fondazione Collegio Carlo Alberto or the Banca d'Italia. 


\section{Introduction}

Since the seminal contributions of Markowitz [Ma] and Tobin [To], meanvariance preferences have been extensively used to model the behavior of economic agents choosing among uncertain prospects and have become one of the workhorses of portfolio selection theory. ${ }^{1}$ These preferences, denoted by $\succeq_{m v}$, assign to an uncertain prospect $f$ the following utility score:

$$
U_{\theta}(f)=\mathrm{E}^{P}[f]-\frac{\theta}{2} \operatorname{Var}^{P}[f],
$$

where $P$ is a given probability measure and $\theta$ is an index of the agent's aversion to variance.

The success of this specification of preferences is due to its analytical tractability and clear intuitive meaning. Mean-variance preferences have, however, a major theoretical drawback: they may fail to be monotone. It may happen that an agent with mean-variance preferences strictly prefers less to more, thus violating one of the most compelling principles of economic rationality. This is especially troublesome in Finance because monotonicity is the crucial assumption on preferences that arbitrage arguments require (see Dybvig and Ross [DR] and Ross [R]).

The lack of monotonicity of mean-variance preferences is a well known problem (see, e.g., Dybvig and Ingersoll [DI] and Jarrow and Madan [JM]) and not a minor one, since it can be (partly) bypassed only under very restrictive assumptions about the statistical distribution of asset returns (see, e.g., Bigelow [Bi]).

The non-monotonicity of mean-variance preferences can be illustrated with a simple example. Consider a mean-variance agent with $\theta=2$. Suppose she has to choose between the two following prospects $f$ and $g$ :

\begin{tabular}{lllll}
\hline States of Nature & $s_{1}$ & $s_{2}$ & $s_{3}$ & $s_{4}$ \\
\hline Probabilities & 0.25 & 0.25 & 0.25 & 0.25 \\
Payoff of $f$ & 1 & 2 & 3 & 4 \\
Payoff of $g$ & 1 & 2 & 3 & 5 \\
\hline
\end{tabular}

Prospect $g$ yields a higher payoff than $f$ in every state. Any rational agent should prefer $g$ to $f$. However, it turns out that our mean-variance agent strictly prefers $f$ to $g$. In fact:

$$
U_{2}(f)=1.25>0.5625=U_{2}(g) .
$$

\footnotetext{
${ }^{1}$ See, e.g., Bodie, Kane, and Marcus [BKM], Britten-Jones [Br], Gibbons, Ross, and Shanken [GRS], Kandel and Stambaugh [KS], and MacKinlay and Richardson [MR].
} 
The reason why monotonicity fails here is fairly intuitive. By choosing $g$ rather than $f$, the payoff in state $s_{4}$ increases by one unit. This additional unit increases the mean payoff, but it also makes the distribution of payoffs more spread out, thus increasing the variance. The increase in the mean is more than compensated by the increase in the variance, and this makes our mean-variance agent worse off.

In this paper we consider the minimal modification of mean-variance preferences needed to overcome their lack of monotonicity. This amended version, based on the variational preferences of Maccheroni, Marinacci, and Rustichini [MMR], is not only sounder from an economic rationality viewpoint, but, being as close as possible to the original, also maintains the basic intuition and tractability of mean-variance preferences.

Specifically, we consider the variational preference $\succeq_{m m v}$ represented by the choice functional

$$
V_{\theta}(f)=\min _{Q}\left\{\mathrm{E}^{Q}[f]+\frac{1}{2 \theta} C(Q \| P)\right\} \quad \forall f \in \mathcal{L}^{2}(P),
$$

where $Q$ ranges over all probability measures with square-integrable density with respect to $P$, and $C(Q \| P)$ is the relative Gini concentration index (or $\chi^{2}$-distance), a concentration index that enjoys properties similar to those of the relative entropy.

The preferences $\succeq_{m m v}$ have the following key properties:

- They are monotone and they agree with mean-variance preferences where the latter are monotone, that is, economically meaningful. ${ }^{2}$

- Their choice functional $V_{\theta}$ is the minimal, and so the most cautious, monotone functional that extends the mean-variance functional $U_{\theta}$ outside its domain of monotonicity.

- The functional $V_{\theta}$ is also the best possible monotone approximation of $U_{\theta}$ : that is, if $V_{\theta}^{\prime}$ is any other monotone extension of $U_{\theta}$ outside its domain of monotonicity, then

$$
\left|V_{\theta}(f)-U_{\theta}(f)\right| \leq\left|V_{\theta}^{\prime}(f)-U_{\theta}(f)\right|
$$

for each prospect $f$.

Moreover:

\footnotetext{
${ }^{2}$ This is the set where $\nabla U_{\theta}$ is positive, called domain of monotonicity of $U_{\theta}$.
} 
- The functional relation between $V_{\theta}$ and $U_{\theta}$ can be explicitly formulated.

- The parameter $\theta$ retains the usual interpretation in terms of uncertainty aversion.

- The functional $V_{\theta}$ preserves second order stochastic dominance.

All these features make the preferences $\succeq_{m m v}$ a natural adjusted version of mean-variance preferences that satisfies monotonicity. For this reason we call them monotone mean-variance preferences.

In view of all this, it is natural to wonder what happens in a portfolio problem à la Markowitz when we use monotone mean-variance preferences in place of standard mean-variance preferences. This is the main subject matter of this paper. Markowitz's well-known optimal allocation rule under mean-variance preferences is:

$$
\alpha_{m v}^{*}=\frac{1}{\theta} \operatorname{Var}^{P}[X]^{-1} \mathrm{E}^{P}[X-\overrightarrow{1} R]
$$

where $\alpha_{m v}^{*}$ is the optimal portfolio of risky assets, $X$ is the vector of gross returns on the risky assets, $R$ is the gross return on the risk-free asset, and $\overrightarrow{1}$ is a vector of $1 \mathrm{~s}$. We show that with monotone mean-variance preferences the optimal allocation rule becomes:

$$
\alpha_{m m v}^{*}=\frac{1}{\theta P(W \leq \kappa)} \operatorname{Var}^{P}[X \mid W \leq \kappa]^{-1} \mathrm{E}^{P}[X-\overrightarrow{1} R \mid W \leq \kappa],
$$

where $W$ is future wealth and $\kappa$ is a constant determined along with $\alpha_{m m v}^{*}$ by solving a suitable system of equations.

Except for a scaling factor, the difference between Markowitz's optimal portfolio $\alpha_{m v}^{*}$ and the above portfolio $\alpha_{m m v}^{*}$ is that in the latter conditional moments of asset returns $\mathrm{E}^{P}[\cdot \mid W \leq \kappa]$ and $\operatorname{Var}^{P}[\cdot \mid W \leq \kappa]$ are used instead of unconditional moments, so that the allocation $\alpha_{m m v}^{*}$ ignores the part of the distribution where wealth is higher than $\kappa$. As a result, a monotone mean-variance agent does not take into account those high payoff states that contribute to increase the mean return, but give an even greater contribution to increase the variance. By doing so, this agent does not incur in violations of monotonicity caused for mean-variance preferences by an exaggerate penalization of "positive deviations from the mean." This is a key feature of monotone mean-variance preferences. We further illustrate this point in Section 6 by showing how this functional avoids some pathological situations in which the more the payoff to an asset is increased in some states, the more a 
mean-variance agent reduces the quantity of it in her portfolio, until in the limit she ends up holding none.

In the last part of the paper we derive a monotone CAPM model based on the above portfolio analysis with our monotone mean-variance preferences. We first show that in our model optimal portfolios satisfy the classic two fund separation principle: (i) portfolios of risky assets optimally held by agents with different degrees of uncertainty aversion are all proportional to each other, (ii) at an optimum the only difference between two agents is the amount of wealth invested in the risk-free asset. This separation has significant theoretical implications because it allows to identify the equilibrium market portfolio with the optimal portfolio of risky assets held by any agent (as in [Sh], [Sh-2] and [To]), and it allows to derive a monotone version of the classic CAPM.

In Section 5 we show that the monotone CAPM that we derive has the empirical tractability of the standard CAPM. Moreover, thanks to monotonicity of the preference functional $V_{\theta}$, in the monotone CAPM there are no arbitrage opportunities. This is a key property of the monotone CAPM and is in stark contrast with what happens with the standard CAPM. In fact, as observed by Dybvig and Ingersoll [DI], the lack of monotonicity of mean-variance preferences generates arbitrage opportunities in the standard CAPM. In turn, these arbitrage opportunities make impossible to have CAPM equilibrium prices of all assets in a complete-markets economy. This is, instead, possible in our arbitrage free monotone CAPM, which can thus be integrated in the classic Arrow-Debreu complete-markets framework.

The paper is organized as follows. Section 2 illustrates in detail monotone mean-variance preferences. Sections 3 and 4 state and solve the portfolio selection problem under the proposed specification of preferences. Section 5 contains the CAPM analysis. Section 6 presents some examples that illustrate the difference between the optimal allocation rule proposed here and Markowitz's. Section 7 concludes. All proofs are collected in the appendices, along with some general results on monotone approximations of concave functionals.

\section{Monotone Mean-Variance Preferences}

We consider a measurable space $(S, \Sigma)$ of states of nature. An uncertain prospect is a $\Sigma$-measurable real valued function $f: S \rightarrow \mathbb{R}$, that is, a stochastic monetary payoff. 
The agent's preferences are described by a binary relation $\succeq$ on a set of uncertain prospects. $[\mathrm{MMR}]$ provides a set of simple behavioral conditions that guarantee the existence of an increasing utility function $u: \mathbb{R} \rightarrow \mathbb{R}$ and a convex uncertainty index $c: \Delta \rightarrow[0, \infty]$ on the set $\Delta$ of all probability measures, such that

$$
f \succeq g \Leftrightarrow \inf _{Q \in \Delta}\left\{\mathrm{E}^{Q}[u(f)]+c(Q)\right\} \geq \inf _{Q \in \Delta}\left\{\mathrm{E}^{Q}[u(g)]+c(Q)\right\}
$$

for all (simple) prospects $f, g$.

Preferences having such a representation are called variational, and two important special cases of variational preferences are the multiple priors preferences of Gilboa and Schmeidler [GS], obtained when $c$ only takes on values 0 and $\infty$, and the multiplier preferences of Hansen and Sargent [HS], obtained when $c(Q)$ is proportional to the relative entropy of $Q$ with respect to a reference probability measure $P{ }^{3}$

Variational preferences satisfy the basic tenets of economic rationality. In particular, they are monotone, that is, given any two prospects $f$ and $g$, we have $f \succeq g$ whenever $f(s) \geq g(s)$ for each $s \in S .{ }^{4}$

For concreteness, given a probability measure $P$ on $(S, \Sigma)$, we consider the set $\mathcal{L}^{2}(P)$ of all square integrable uncertain prospects. A relation $\succeq_{m v}$ on $\mathcal{L}^{2}(P)$ is a mean-variance preference if it is represented by the choice functional

$$
U_{\theta}(f)=\mathrm{E}^{P}[f]-\frac{\theta}{2} \operatorname{Var}^{P}[f] \quad \forall f \in \mathcal{L}^{2}(P),
$$

for some $\theta>0$.

The subset $\mathcal{G}_{\theta}$ of $\mathcal{L}^{2}(P)$ where the Gateaux differential of $U_{\theta}$ is positive is called domain of monotonicity of $U_{\theta}$. The preference $\succeq_{m v}$ is monotone on the set $\mathcal{G}_{\theta}$, which has the following properties.

Lemma 1 The set $\mathcal{G}_{\theta}$ is convex, closed, and

$$
\mathcal{G}_{\theta}=\left\{f \in \mathcal{L}^{2}(P): f-\mathrm{E}^{P}[f] \leqslant \frac{1}{\theta}\right\}
$$

Moreover, for all $f \notin \mathcal{G}_{\theta}$ and every $\varepsilon>0$ there exists $g \in \mathcal{L}^{2}(P)$ that is $\varepsilon$-close to $f$ (i.e., $|f(s)-g(s)|<\varepsilon$ for all $s \in S$ ), and such that $g>f$ and $U_{\theta}(g)<U_{\theta}(f)$.

\footnotetext{
${ }^{3}$ The relative entropy of $Q$ given $P$ is $\mathrm{E}^{P}\left[\frac{d Q}{d P} \ln \frac{d Q}{d P}\right]$ if $Q \ll P$ and $\infty$ otherwise.

${ }^{4}$ In the special case in which $u$ is linear, some recent finance papers (e.g., Filipovic and Kupper [FK] and Kupper and Cheridito $[\mathrm{KC}]$ ) call monetary utility functions the functionals representing variational preferences.
} 
The domain of monotonicity has thus nice properties. More importantly, the last part of the lemma shows why $\mathcal{G}_{\theta}$ is where the mean-variance preference $\succeq_{m v}$ is economically meaningful. In fact, it says that if we take any prospect $f$ outside $\mathcal{G}_{\theta}$, then in every its neighborhood, however small, there is at least a prospect $g$ that is statewise better than $f$, but ranked by $\succeq_{m v}$ below $f$.

The mean-variance preference $\succeq_{m v}$ thus exhibits irrational non-monotone behavior in every neighborhood, however small, of prospects $f$ outside $\mathcal{G}_{\theta}$. For this reason $\succeq_{m v}$ has no economic meaning outside $\mathcal{G}_{\theta}$.

It can be shown that the restriction of $\succeq_{m v}$ to $\mathcal{G}_{\theta}$ is a variational preference, and

$$
U_{\theta}(f)=\min _{Q \in \Delta^{2}(P)}\left\{\mathrm{E}^{Q}[f]+\frac{1}{2 \theta} C(Q \| P)\right\} \quad \forall f \in \mathcal{G}_{\theta},
$$

where $\Delta^{2}(P)$ is the set of all probability measures with square-integrable density with respect to $P$, and

$$
C(Q \| P)= \begin{cases}\mathrm{E}^{P}\left[\left(\frac{d Q}{d P}\right)^{2}\right]-1 & \text { if } Q \ll P \\ +\infty & \text { otherwise }\end{cases}
$$

is the relative Gini concentration index (or $\chi^{2}$-distance).$^{5}$

This suggests to call monotone mean-variance preference the relation $\succeq_{m m v}$ on $\mathcal{L}^{2}(P)$ represented by the choice functional

$$
V_{\theta}(f)=\min _{Q \in \Delta^{2}(P)}\left\{\mathrm{E}^{Q}[f]+\frac{1}{2 \theta} C(Q \| P)\right\} \quad \forall f \in \mathcal{L}^{2}(P) .
$$

Our first result is the following: ${ }^{6}$

Theorem 2 The functional $V_{\theta}: \mathcal{L}^{2}(P) \rightarrow \mathbb{R}$ defined by (3) is the minimal monotone functional on $\mathcal{L}^{2}(P)$ such that $V_{\theta}(g)=U_{\theta}(g)$ for all $g \in \mathcal{G}_{\theta}$; that is,

$$
V_{\theta}(f)=\sup \left\{U_{\theta}(g): g \in \mathcal{G}_{\theta} \text { and } g \leqslant f\right\} \quad \forall f \in \mathcal{L}^{2}(P) .
$$

Moreover, $V_{\theta}(f) \geq U_{\theta}(f)$ for each $f \in \mathcal{L}^{2}(P)$.

\footnotetext{
${ }^{5}$ Along with the Shannon entropy, the Gini index is the most classic concentration index. For discrete distributions it is given by $\sum_{i=1}^{n} Q_{i}^{2}-1$, and $C(Q \| P)$ is its continuous and relative version. We refer to [LV] for a comprehensive study of concentration indices.

${ }^{6}$ The proof of this theorem builds on a general result on the minimal monotone functional that dominates a concave functional on an ordered Banach space, which we prove in Appendix A (Proposition 12).
} 
The functional $V_{\theta}$ is concave, continuous, and in view of this theorem it has the following fundamental properties:

(i) $V_{\theta}$ coincides with the mean-variance choice functional $U_{\theta}$ on its domain of monotonicity $\mathcal{G}_{\theta}$.

(ii) $V_{\theta}$ is the minimal monotone extension of $U_{\theta}$ outside the domain of monotonicity $\mathcal{G}_{\theta}$, and so it is the most cautious monotone adjustment of the mean-variance choice functional.

(iii) $V_{\theta}$ is the best possible monotone approximation of $U_{\theta}$ : if $V_{\theta}^{\prime}$ is any other monotone extension of $U_{\theta}$ outside the domain of monotonicity $\mathcal{G}_{\theta}$, then $V_{\theta}^{\prime}(f) \geq V_{\theta}(f) \geq U_{\theta}(f)$ and so

$$
\left|V_{\theta}(f)-U_{\theta}(f)\right| \leq\left|V_{\theta}^{\prime}(f)-U_{\theta}(f)\right| \quad \forall f \in \mathcal{L}^{2}(P) .
$$

Next theorem shows explicitly the functional relation between $V_{\theta}$ and $U_{\theta}$.

Theorem 3 Let $f \in \mathcal{L}^{2}(P)$. Then:

$$
V_{\theta}(f)= \begin{cases}U_{\theta}(f) & \text { if } f \in \mathcal{G}_{\theta}, \\ U_{\theta}\left(f \wedge \kappa_{f}\right) & \text { else, }\end{cases}
$$

where

$$
\kappa_{f}=\max \left\{t \in \mathbb{R}: f \wedge t \in \mathcal{G}_{\theta}\right\} .
$$

A monotone mean-variance agent can thus be regarded as still using the mean-variance functional $U_{\theta}$ even in evaluating prospects outside the domain of monotonicity $\mathcal{G}_{\theta}$. In this case, however, the agent no longer considers the original prospects, but rather their truncations at $\kappa_{f}$, the largest constant $t$ such that $f \wedge t$ belongs to $\mathcal{G}_{\theta}$.

Besides depending on the given prospect $f$, the constant $\kappa_{f}$ also depends on the parameter $\theta$. Corollary 16 in Appendix B shows that $\kappa_{f}$ decreases as $\theta$ increases, and it is the unique solution of the equation $\mathrm{E}^{P}\left[(f-\kappa)^{-}\right]=1 / \theta$.

Given two preferences over uncertain prospects, we say that $\succeq^{1}$ is more uncertainty averse than $\succeq^{2}$ if and only if

$$
f \succeq^{1} c \Longrightarrow f \succeq^{2} c
$$

for all $f \in \mathcal{L}^{2}(P)$ and $c \in \mathbb{R}$. That is, Agent 1 is more uncertainty averse than Agent 2 if, whenever Agent 1 prefers the uncertain prospect $f$ to a sure payoff $c$, so does Agent $2 .^{7}$

\footnotetext{
${ }^{7}$ We refer the interested reader to [MMR] for a discussion of this notion, and its interpretation in terms of risk aversion and ambiguity aversion (not mentioned here in order to keep the paper focused).
} 
A mean-variance preference $\succeq_{m v}^{\theta}$ is more uncertainty averse than another mean-variance preference $\succeq_{m v}^{\gamma}$ if and only if $\theta>\gamma$. Thus, $\theta$ can be interpreted as an uncertainty aversion coefficient.

The next result, a variation of [MMR, Cor. 21], shows that the same is true for monotone mean-variance preferences.

Proposition 4 The preference $\succeq_{m m v}^{\theta}$ is more uncertainty averse than $\succeq_{m m v}^{\gamma}$ if and only if $\theta>\gamma$.

We conclude this section by observing that, unlike $U_{\theta}$, the preference functional $V_{\theta}$ preserves second order stochastic dominance $\left(\succeq_{S S D}\right){ }^{8}$ This is a further proof of the sounder economic meaning of monotone mean-variance preferences relative to mean-variance ones.

Theorem 5 Let $f, g \in \mathcal{L}^{2}(P)$. If $f \succeq_{S S D} g$, then $V_{\theta}(f) \geq V_{\theta}(g)$.

Summing up, the monotone choice functional $V_{\theta}$ provides a natural adjustment of the mean-variance choice functional. It also has the remarkable feature of involving, like multiplier preferences $([\mathrm{HS}])$, a classic concentration index. This ensures to $V_{\theta}$ a good analytical tractability, as the next sections show.

\section{The Portfolio Selection Problem}

We consider the one-period allocation problem of an agent who has to decide how to invest a unit of wealth at time 0 , dividing it among $n+1$ assets. The first $n$ assets are risky, while the $(n+1)$-th is risk-free. The gross return on the $i$-th asset after one period is denoted by $X_{i}$. The $(n \times 1)$ vector of the returns on the first $n$ assets is denoted by $X$ and the $(n \times 1)$ vector of portfolio weights, indicating the fraction of wealth invested in each of the risky assets, is denoted by $\alpha$. The return on the $(n+1)$-th asset is risk-free and equal to a constant $R$.

The end-of-period wealth $W_{\alpha}$ induced by a choice of $\alpha$ is given by:

$$
W_{\alpha}=R+\alpha \cdot(X-\overrightarrow{1} R) .
$$

We assume that there are no frictions of any kind: securities are perfectly divisible; there are no transaction costs or taxes; agents are price-takers, in

\footnotetext{
${ }^{8}$ Recall that $f \succeq_{S S D} g$ iff $\mathrm{E}^{P}\left[(f-t)^{-}\right] \leq \mathrm{E}^{P}\left[(g-t)^{-}\right]$for all $t \in \mathbb{R}$. We refer to Dana [Da], to which the proof of Theorem 5 is inspired, for references on second order stochastic dominance.
} 
that they believe that their choices do not affect the distribution of asset returns; there are no institutional restrictions, so that agents are allowed to buy, sell or short sell any desired amount of any security. ${ }^{9}$ As a result, $\alpha$ can be chosen in $\mathbb{R}^{n}$.

We adopt $\succeq_{m m v}$ as a specification of preferences, and so portfolios are ranked according to the preference functional:

$$
V_{\theta}\left(W_{\alpha}\right)=\min _{Q \in \Delta^{2}(P)}\left(\mathrm{E}^{Q}\left[W_{\alpha}\right]+\frac{1}{2 \theta} C(Q \| P)\right),
$$

where $P$ is the reference probability measure. Hence, the portfolio problem can be written as:

$$
\max _{\alpha \in \mathbb{R}^{n}} \min _{Q \in \Delta^{2}(P)}\left(\mathrm{E}^{Q}\left[W_{\alpha}\right]+\frac{1}{2 \theta} C(Q \| P)\right) .
$$

Notice that, if the agent's initial wealth is $w>0$, then her end-of-period wealth is $w W_{\alpha}$, therefore she solves the problem

$$
\max _{\alpha \in \mathbb{R}^{n}} \min _{Q \in \Delta^{2}(P)}\left(\mathrm{E}^{Q}\left[w W_{\alpha}\right]+\frac{1}{2 \theta} C(Q \| P)\right)
$$

which - dividing the argument by $w$ - reduces to (6) up to replacement of $\theta$ with $\theta w$.

\section{The Optimal Portfolio}

In this section we give a solution to the portfolio selection problem outlined in the previous section. The characterization of the optimal portfolio is given by the following theorem. ${ }^{10}$

Theorem 6 The vector $\alpha^{*} \in \mathbb{R}^{n}$ is a solution of the portfolio selection problem (6) if and only if there exists $\kappa^{*} \in \mathbb{R}$ such that $\left(\alpha^{*}, \kappa^{*}\right)$ satisfies the system of equations:

$$
\left\{\begin{array}{l}
\theta P\left(W_{\alpha} \leq \kappa\right) \operatorname{Var}^{P}\left[X \mid W_{\alpha} \leq \kappa\right] \alpha=\mathrm{E}^{P}\left[X-\overrightarrow{1} R \mid W_{\alpha} \leq \kappa\right] \\
\mathrm{E}^{P}\left[\left(W_{\alpha}-\kappa\right)^{-}\right]=1 / \theta .
\end{array}\right.
$$

\footnotetext{
${ }^{9}$ This assumption can be weakened, by simply requiring that at an optimum institutional restrictions are not binding.

${ }^{10} E^{P}\left[\cdot \mid W_{\alpha} \leq \kappa\right]$ and $\operatorname{Var}^{P}\left[\cdot \mid W_{\alpha} \leq \kappa\right]$ are the expectation and variance conditional on the event $\left\{W_{\alpha} \leq \kappa\right\}$. Note that $\operatorname{Var}^{P}\left[\cdot \mid W_{\alpha} \leq \kappa\right]$ is an $(n \times n)$ matrix.
} 
As observed in Section 2, the second displayed equation guarantees that $\kappa^{*}$ is the largest constant such that $W_{\alpha^{*}} \wedge \kappa^{*}$ belongs to the domain of monotonicity of the mean-variance functional $U_{\theta}$. The optimal portfolio $\alpha^{*}$ is thus determined along with the threshold $\kappa^{*}$ by solving a system of $n+1$ equations in $n+1$ unknowns.

Although it is not generally possible to find explicitly a solution of the above system of equations, numerical calculation with a standard equation solver is straightforward: given an initial guess $(\alpha, \kappa)$, one is able to calculate the first two moments of the conditional distribution of wealth; if the moments thus calculated, together with the initial guess $(\alpha, \kappa)$, satisfy the system of equations, then $(\alpha, \kappa)=\left(\alpha^{*}, \kappa^{*}\right)$ and numerical search stops; otherwise, the search procedure continues with a new initial guess for the parameters. ${ }^{11}$ In the next section we will solve in this way few simple portfolio problems in order to illustrate some features of the model.

The optimal allocation rule of Theorem 6 is easily compared to the rule that would obtain in a classic Markowitz's setting. In the traditional meanvariance model we would have:

$$
\alpha^{*}=\frac{1}{\theta}\left[\operatorname{Var}^{P}[X]\right]^{-1} \mathrm{E}^{P}[X-\overrightarrow{1} R] .
$$

The monotone mean-variance model yields:

$$
\alpha^{*}=\frac{1}{\theta P\left(W_{\alpha^{*}} \leq \kappa^{*}\right)} \operatorname{Var}^{P}\left[X \mid W_{\alpha^{*}} \leq \kappa^{*}\right]^{-1} \mathrm{E}^{P}\left[X-\overrightarrow{1} R \mid W_{\alpha^{*}} \leq \kappa^{*}\right] .
$$

Relative to Markowitz's optimal allocation (7), here the unconditional mean and variance of the vector of returns $X$ are replaced by a conditional mean and a conditional variance, both calculated by conditioning on the event $\left\{W_{\alpha^{*}} \leq \kappa^{*}\right\}$. Furthermore a scaling factor is introduced, which is inversely proportional to the probability of not exceeding the threshold $\kappa^{*}$.

Roughly speaking, when computing the optimal portfolio we ignore that part of the distribution where wealth is higher than $\kappa^{*}$. To see why it is optimal to ignore the part of the distribution where one obtains the highest returns, recall the example of non-monotonicity of mean-variance illustrated in the Introduction. In that example, high payoffs were increasing the variance more than the mean, thus leading the mean-variance agent to irrationally prefer a strictly smaller prospect. With monotone mean-variance preferences, this kind of behavior is avoided by artificially setting the probability of some high payoff states equal to zero. In our portfolio selection problem we set the probability of the event $\left\{W_{\alpha^{*}}>\kappa^{*}\right\}$ equal to zero.

\footnotetext{
${ }^{11} \mathrm{~A} \mathrm{R}$ (S-Plus) routine to calculate the optimal portfolio in an economy with finitely many states of nature is available upon request.
} 
When there is only one risky asset, the optimal quantities $\alpha_{m m v}^{*}$ and $\alpha_{m v}^{*}$ prescribed, respectively, by our model and by the mean-variance model can be compared by means of the following result.

Proposition 7 Suppose that $S$ is finite, with $P(s)>0$ for all $s \in S$, and that there is only one risky asset. Then, either

$$
\alpha_{m m v}^{*} \geq \alpha_{m v}^{*} \geq 0
$$

or

$$
\alpha_{m m v}^{*} \leq \alpha_{m v}^{*} \leq 0 .
$$

If, in addition, $P\left(W_{\alpha_{m m v}^{*}}>\kappa^{*}\right)>0$, then:

$$
\begin{array}{ll}
\alpha_{m m v}^{*}>\alpha_{m v}^{*} & \text { if } \alpha_{m m v}^{*}>0, \\
\alpha_{m m v}^{*}<\alpha_{m v}^{*} & \text { if } \alpha_{m m v}^{*}<0 .
\end{array}
$$

That is, an investor with monotone mean-variance preferences always holds a portfolio which is more leveraged than the portfolio held by a meanvariance investor. If she buys a positive quantity of the risky asset, this is greater than the quantity that would be bought by a mean-variance investor; on the contrary, if she sells the risky asset short, she sells more than a mean-variance investor would do. This kind of behavior will be thoroughly illustrated by the examples in the next section: the intuition behind it is that in some cases a favorable investment opportunity is discarded by a mean-variance investor because of non-monotonicity of her preferences, while a monotone mean-variance investor exploits the opportunity, thus taking a more leveraged position.

\section{Monotone CAPM}

In this section we show how the standard CAPM analysis can be carried out in the monotone mean-variance setup.

We begin by establishing a two-funds separation result, which shows that agents' optimal investment choices can be done in two stages: first agents decide the amount of wealth to invest in the risk-free asset; then, they decide how to allocate the remaining wealth among the risky assets. The outcome of this second decision is the same for all agents, regardless of their initial wealth or aversion to uncertainty.

Proposition 8 Let $\theta, \gamma>0$. If $\left(\alpha^{\theta}, \kappa^{\theta}\right)$ solves the portfolio selection problem (6) for an investor with uncertainty aversion $\theta$, then $\left(\frac{\theta}{\gamma} \alpha^{\theta}, \frac{\theta}{\gamma} \kappa^{\theta}+\left(1-\frac{\theta}{\gamma}\right) R\right)$ solves it for an investor with uncertainty aversion $\gamma$. 
Given a $\theta>0$ with $\alpha^{\theta} \cdot \overrightarrow{1}>0$, define $m=\theta \alpha^{\theta} \cdot \overrightarrow{1}$. Hence,

$$
\alpha^{m}=\frac{\alpha^{\theta}}{\alpha^{\theta} \cdot \overrightarrow{1}}
$$

and Proposition 8 guarantees that $m$ and $\alpha^{m}$ do not depend on the choice of $\theta$. The equality $\alpha^{m} \cdot \overrightarrow{1}=1$ implies that $\alpha^{m}$ is the portfolio held by an investor who does not invest any of her wealth in the risk-free asset. Following the majority of the literature, we call $\alpha^{m}$ the market portfolio and denote by $X_{m}=\alpha^{m} \cdot X$ its payoff. In particular, $W_{\alpha^{m}}=R+\alpha^{m} \cdot(X-\overrightarrow{1} R)=X_{m}$.

In an economy consisting of monotone-mean variance agents, all investors hold a portfolio of risky assets proportional to the market portfolio. Specifically, an investor with uncertainty aversion $\theta$ will invest $m / \theta$ in the market portfolio and the rest of her wealth in the risk free asset. Like in the standard mean-variance setting, also here the amount of wealth invested in the market portfolio only depends on the coefficient $\theta$ of the agent.

All this has strong empirical implications. From market data - more precisely, by observing the market values of the assets in the economy - it is possible to determine the equilibrium composition of the market portfolio $\alpha^{m}$. Once we know the equilibrium $\alpha^{m}$, and so its equilibrium payoff $X_{m}$, thanks to the next result we can find the values of $m$ and $\kappa^{m}$ by solving a system of equations with observable coefficients. ${ }^{12}$

Proposition 9 The pair $\left(x^{*}, y^{*}\right) \equiv\left(m, \kappa^{m}\right)$ solves the following system of equations

$$
\left\{\begin{array}{l}
P\left(X_{m} \leq y\right) \operatorname{Var}^{P}\left[X_{m} \mid X_{m} \leq y\right] x=\mathrm{E}^{P}\left[X_{m}-R \mid X_{m} \leq y\right] \\
\mathrm{E}^{P}\left[\left(X_{m}-y\right)^{-}\right]=1 / x
\end{array}\right.
$$

The knowledge of $m$ and $\kappa^{m}$ makes it possible to determine the equilibrium pricing kernel $\nabla V_{m}\left(X_{m}\right)$, which will become very important momentarily when discussing the monotone CAPM. To see why this is the case, we need the following lemma, which gives some properties of $\nabla V_{m}\left(X_{m}\right)$.

Lemma 10 The quantity $\nabla V_{m}\left(X_{m}\right)$ has the following properties:

(i) $\nabla V_{m}\left(X_{m}\right)=m\left(X_{m}-\kappa^{m}\right)^{-}=\nabla V_{\theta}\left(W_{\alpha^{\theta}}\right)$ for all $\theta>0$.

\footnotetext{
${ }^{12}$ Notice that, like in the standard mean-variance setting, it can also be shown that the uncertainty aversion coefficient $m$ is a mean of the uncertainty aversion coefficients of the agents. Specifically, $m=\rho\left(\sum_{j}\left(\theta^{j}\right)^{-1}\right)^{-1}$ where $\theta^{j}$ is the uncertainty aversion coefficient of agent $j$ and $\rho$ is the market value of all assets.
} 
(ii) $\mathrm{E}^{P}\left[X_{i} \nabla V_{m}\left(X_{m}\right)\right]=R$ for all $i=1, \ldots, n$.

(iii) $\mathrm{E}^{P}\left[\nabla V_{m}\left(X_{m}\right)\right]=1$.

By property (i) of this lemma, once we know the values of $m$ and $\kappa^{m}$ we can determine the value of $\nabla V_{m}\left(X_{m}\right)$ via the equation $m\left(X_{m}-\kappa^{m}\right)^{-}$. The value of $\nabla V_{m}\left(X_{m}\right)$ can thus be determined from market data. To ease notation, in what follows we set $\nabla V \equiv \nabla V_{m}\left(X_{m}\right)$.

Lemma 10 also makes it possible to derive our monotone version of the CAPM. In fact, by Lemma 10.(ii), $\mathrm{E}^{P}\left[X_{m} \nabla V\right]=\alpha^{m} \cdot \mathrm{E}^{P}[X \nabla V]=R$. Together with Lemma 10.(iii), this implies

$$
\operatorname{Cov}^{P}\left[X_{i}, \nabla V\right]=R-\mathrm{E}^{P}\left[X_{i}\right] \text { and } \operatorname{Cov}^{P}\left[X_{m}, \nabla V\right]=R-\mathrm{E}^{P}\left[X_{m}\right],
$$

which proves the following theorem, the main result of this section.

Theorem 11 (Monotone CAPM) Let $X_{m}$ be defined as above. Then,

$$
\mathrm{E}^{P}\left[X_{i}\right]-R=\beta_{i}\left(\mathrm{E}^{P}\left[X_{m}\right]-R\right), \quad \forall i=1, \ldots, n,
$$

where

$$
\beta_{i}=\frac{\operatorname{Cov}^{P}\left[X_{i}, \nabla V\right]}{\operatorname{Cov}^{P}\left[X_{m}, \nabla V\right]} .
$$

Theorem 11 gives our monotone CAPM, with security market line (8), and shows its key theoretical and empirical features.

On the theoretical side, the pricing rule delivered by our CAPM is arbitrage free: there are no portfolios with strictly negative prices and positive final payoffs. In fact, let $Y_{i}$ the end-of-period payoff per share of asset $i$ and $p_{i}$ its current price. Then, $X_{i}=Y_{i} / p_{i}$ and (8) becomes

$$
\frac{\mathrm{E}^{P}\left[Y_{i}\right]}{p_{i}}-R=\frac{1}{p_{i}} \frac{\operatorname{Cov}^{P}\left[Y_{i}, \nabla V\right]}{\operatorname{Cov}^{P}\left[X_{m}, \nabla V\right]}\left(\mathrm{E}^{P}\left[X_{m}\right]-R\right)
$$

This delivers the pricing rule:

$$
p_{i}=\frac{1}{R} \mathrm{E}^{P}\left[Y_{i} \nabla V\right] .
$$

This pricing rule is a positive linear functional. For, the price of a portfolio consisting of $q_{i}$ shares of asset $i$ is

$$
\sum_{i=1}^{n} q_{i} p_{i}=\frac{1}{R} \mathrm{E}^{P}\left[\left(\sum_{i=1}^{n} q_{i} Y_{i}\right) \nabla V\right]
$$


which is positive as long as $\sum_{i=1}^{n} q_{i} Y_{i}$ is positive $\left(\nabla V\right.$ is positive because $V_{m}$ is monotone).

The absence of arbitrage opportunities in our monotone CAPM is a key theoretical feature of our model. As observed in the Introduction, this is in stark contrast with their presence in the standard CAPM model, caused by the lack of monotonicity of mean-variance preferences. This was observed by Dybvig and Ingersoll [DI], who show that if $X_{m} \notin \mathcal{G}_{m}$ and the market is complete, then the pricing rule obtained from standard CAPM is not a positive linear functional, and it thus allows arbitrages.

Inter alia, this means that, differently from the standard CAPM, our monotone CAPM pricing rule can be integrated in a standard Arrow-Debreu complete-markets economy, with all assets in such an economy priced in equilibrium according to our CAPM.

On the empirical side, our monotone CAPM model can be fully analyzed from market data. First observe that the values of the betas (9) can be derived from market data because we just observed that, besides those of $X_{i}$, also the values of $X_{m}$ and $\nabla V$ can be determined from market data.

Second, Theorem 11 suggests that, by regressing the excess returns to the single assets on the excess return to the market portfolio, the empirical betas of the single assets can be estimated by instrumental variables, using $\nabla V$ as an instrument. In fact, define $\varepsilon_{i}=X_{i}-R-\beta_{i}\left(X_{m}-R\right)$, so that:

$$
X_{i}=R+\beta_{i}\left(X_{m}-R\right)+\varepsilon_{i} .
$$

By Lemma 10.(ii), $\nabla V$ is easily seen to be orthogonal to $\varepsilon_{i}$, and so $\nabla V$ can be used as an instrument.

We cannot use, instead, ordinary least squares because, in general, $X_{m}-R$ is not orthogonal to $\varepsilon_{i}$. For:

$$
\begin{aligned}
\mathrm{E}^{P}\left[\varepsilon_{i}\left(X_{m}-R\right)\right] & =\mathrm{E}^{P}\left[\left(X_{i}-R\right)\left(X_{m}-R\right)\right]-\beta_{i} \mathrm{E}^{P}\left[\left(X_{m}-R\right)^{2}\right] \\
& =\mathrm{E}^{P}\left[\left(X_{i}-R\right)\left(X_{m}-R\right)\right]-\frac{\operatorname{Cov}^{P}\left[\nabla V, X_{i}\right]}{\operatorname{Cov}^{P}\left[\nabla V, X_{m}\right]} \mathrm{E}^{P}\left[\left(X_{m}-R\right)^{2}\right] .
\end{aligned}
$$

Summing up, the monotone CAPM is arbitrage free and its betas can be inferred from market data. The monotone CAPM has thus a sounder theoretical foundation than the standard CAPM, while retaining its remarkable empirical tractability. 


\section{Some Examples}

In this section we present three simple examples to illustrate the optimal portfolio rule we derived above. In every example there are five possible states of Nature. Each of them obtains with a probability $P\left(s_{i}\right)$ that remains fixed throughout the examples. In all examples we also set $\theta=10$.

Example 1 is a case in which our model and the traditional mean-variance model deliver the same optimal composition of the portfolio. This is not interesting per se, but it serves as a benchmark and it helps to introduce Example 2, where the two optimal portfolios differ. In Example 1 there is only one risky asset, whose gross return is denoted by $X_{1}$ and is reported in the next table, and a risk-free asset, whose gross return $R$ is equal to 1 across all states. In this example, the optimal portfolio $\alpha_{m m v}^{*}$ calculated according to our rule is equal to the mean-variance optimal portfolio $\alpha_{m v}^{*}$. $W_{m v}$ and $W_{m m v}$ represent the overall return to the two optimal portfolios for each state of the world. The table also displays the value of the constant $\kappa^{*}$ at which it is optimal to truncate the distribution of the return to the portfolio of risky assets.

\begin{tabular}{lllllll}
\hline & $P\left(s_{i}\right)$ & $P\left(s_{i} \mid W_{m m v} \leq \kappa^{*}\right)$ & $R$ & $X_{1}$ & $W_{m v}$ & $W_{m m v}$ \\
\hline$s_{1}$ & 0.1 & 0.1 & 1 & 0.97 & 0.9375 & 0.9375 \\
$s_{2}$ & 0.2 & 0.2 & 1 & 0.99 & 0.9791 & 0.9791 \\
$s_{3}$ & 0.4 & 0.4 & 1 & 1.01 & 1.0208 & 1.0208 \\
$s_{4}$ & 0.2 & 0.2 & 1 & 1.03 & 1.0620 & 1.0620 \\
$s_{5}$ & 0.1 & 0.1 & 1 & 1.05 & 1.1041 & 1.1041 \\
\hline \multicolumn{7}{l}{$\alpha_{m v}^{*}=2.083$} \\
\hline \multicolumn{7}{l}{$\alpha_{m m v}^{*}=2.083$} \\
\hline
\end{tabular}

Example 2 is a slight modification of Example 1. We increase the payoff to the risky asset in state $s_{5}$ from 1.05 to 1.10 , leaving everything else unchanged. The effect of this change is an increase in both the mean and the variance of $X_{1}$, the payoff to the risky asset. The optimal behavior according to the mean-variance model is to reduce the fraction of wealth invested in the risky asset from 2.083 to 1.3574 . According to our model it is also optimal to decrease the position in the risky asset, but less, from 2.083 to 1.8382 . 


\begin{tabular}{lllllll}
\hline & $P\left(s_{i}\right)$ & $P\left(s_{i} \mid W_{m m v} \leq \kappa^{*}\right)$ & $R$ & $X_{1}$ & $W_{m v}$ & $W_{m m v}$ \\
\hline$s_{1}$ & 0.1 & 0.1111 & 1 & 0.97 & 0.9592 & 0.9448 \\
$s_{2}$ & 0.2 & 0.2222 & 1 & 0.99 & 0.9864 & 0.9816 \\
$s_{3}$ & 0.4 & 0.4444 & 1 & 1.01 & 1.0135 & 1.0183 \\
$s_{4}$ & 0.2 & 0.2222 & 1 & 1.03 & 1.0407 & 1.0551 \\
$s_{5}$ & 0.1 & 0 & 1 & 1.10 & 1.1357 & 1.1838 \\
\hline \multicolumn{2}{l}{$\alpha_{m v}^{*}=1.3574$} \\
\hline \multicolumn{2}{l}{$\alpha_{m m v}^{*}=1.8382$} & $\kappa^{*}=1.1213$ \\
\hline
\end{tabular}

In both cases the optimal behavior might seem puzzling at a first sight: when the payoff of an asset increases in one state, it is optimal to hold less of that asset. This behavior can be understood by looking at the distributions of the overall return in the two tables. By reducing the fraction of wealth invested in the risky asset, the overall return increases in the states where the risky asset pays less than the risk-free asset. On the contrary, the overall return decreases in the states where the risky asset pays more than the riskfree asset. In state $s_{5}$, however, the effect of this decrease is compensated by the fact that we have raised the payoff to the risky asset from 1.05 to 1.10. Hence, by reducing the amount of wealth invested in the risky asset, the investor gives up some of the extra payoff received in state $s_{5}$ in order to guarantee himself a higher overall return in the states where the risky asset has a low payoff. The problem with this kind of behavior is that it can become pathological with mean-variance preferences. The next table shows what happens if we further increase the payoff in state $s_{5}$.

\begin{tabular}{llllllll}
\hline$X_{1}\left(s_{5}\right)$ & 1.05 & 1.10 & 1.15 & 1.20 & 1.50 & 2 & 3 \\
\hline$\alpha_{m v}^{*}$ & 2.0830 & 1.3574 & 0.9174 & 0.6747 & 0.2465 & 0.1175 & 0.0572 \\
$\alpha_{m m v}^{*}$ & 2.0830 & 1.8382 & 1.8382 & 1.8382 & 1.8382 & 1.8382 & 1.8382 \\
\hline
\end{tabular}

The more we increase the payoff in state $s_{5}$, the more the mean-variance optimal fraction $\alpha_{m v}^{*}$ of wealth invested in the risky asset decreases, until it goes to zero when the payoff in state $s_{5}$ becomes very large. In our model this does not happen. At first $\alpha_{m m v}^{*}$ decreases, but it then stops decreasing and it remains fixed at the same value, though the payoff in state $s_{5}$ is further increased. The reason why this happens is that, once probabilities have been optimally reassigned to states and a zero probability has been assigned to state $s_{5}$, any further increases of the payoff in $s_{5}$ are disregarded and have no influence on the formation of the optimal portfolio. 
Example 3 is slightly more complicated. Everything is as in Example 2, but a second risky asset is added. The payoff to this new asset, denoted by $X_{2}$, is high in the states where $X_{1}$ is low and low where $X_{1}$ is high.

\begin{tabular}{|c|c|c|c|c|c|c|c|}
\hline & $P\left(s_{i}\right)$ & $P\left(s_{i} \mid W_{m m v} \leq \kappa^{*}\right)$ & $R$ & $X_{1}$ & $X_{2}$ & $W_{m v}$ & $W_{m m v}$ \\
\hline$s_{1}$ & 0.1 & 0.1111 & 1 & 0.97 & 1.05 & 1.002 & 1.0231 \\
\hline$s_{2}$ & 0.2 & 0.2222 & 1 & 0.99 & 1.00 & 0.9833 & 0.9570 \\
\hline$s_{3}$ & 0.4 & 0.4444 & 1 & 1.01 & 0.99 & 1.0061 & 1.0125 \\
\hline$s_{4}$ & 0.2 & 0.2222 & 1 & 1.03 & 0.99 & 1.0393 & 1.0985 \\
\hline$s_{5}$ & 0.1 & 0 & 1 & 1.10 & 0.99 & 1.1556 & 1.3994 \\
\hline \multicolumn{8}{|c|}{$\alpha_{m v}^{*}=(1.6613,1.0495)$} \\
\hline \multicolumn{3}{|c|}{$\alpha_{m m v}^{*}=(4.2989,3.0423)$} & \multicolumn{5}{|c|}{$\kappa^{*}=1.1316$} \\
\hline
\end{tabular}

Also in this case the optimal portfolios suggested by our model and by the traditional model are different. To get an intuitive idea of what is happening, note that, although the market is still arbitrage-free, asset 2 allows to hedge away almost completely the risks taken by investing in asset 1 . Consider for example a portfolio formed by 0.5 units of asset 1 and 0.5 units of asset 2 . Its payoffs in the five states are collected in the following vector:

$$
(1.01,0.995,1,1.01,1.045)
$$

A qualitative inspection of this payoff vector reveals that in state $s_{2}$ this portfolio pays off slightly less than the risk-free asset, while in all other states it pays off more and in some states considerably more. Roughly speaking, if it was not for the slightly low payoff in state $s_{2}$, there would be an arbitrage opportunity because the portfolio would pay off more than the risk-free asset in every state. As a consequence, we would expect an optimal portfolio rule to exploit this favorable configuration of payoffs by prescribing to take a highly levered position. As reported in the last table, according to our model it is optimal to take a highly levered position in the risky assets in order to exploit this opportunity, at the cost of facing a low payoff in state $s_{2}$. In contrast, with the mean-variance model the optimal portfolio is much less aggressive, and the investor is overly concerned with the unique state in which the payoff is lower than the payoff to the risk-free asset.

\section{Conclusions}

We have derived a portfolio allocation rule using a corrected version of the mean-variance principle, which avoids the problem of non-monotonicity. In 
the cases where mean-variance preferences are well-behaved (i.e., monotone) the optimal portfolios suggested by our rule do not differ from standard mean-variance efficient portfolios.

The important property of separability in two funds still holds in our setting, and this allows to derive a monotone CAPM that retains the empirical tractability of the standard CAPM, but, unlike the latter, is arbitrage free.

We close by observing that Maccheroni, Marinacci, and Rustichini [MMR-2] recently extended [MMR] to a dynamic setting, and for this reason we expect that also our analysis can be extended to an intertemporal framework. This will be the subject of future research, along with an empirical analysis of the monotone CAPM.

\section{A Monotone Fenchel Duality}

In this section we recall some important definitions of Convex Analysis, and we prove some properties of monotone extensions of concave functionals that pave the way for the proof of Theorem 2 .

Let $(E,\|\cdot\|, \geqslant)$ be an ordered Banach space, i.e., an ordered vector space endowed with a Banach norm such that the positive cone $E_{+}$is closed and generates $E$. Denote by $E^{\prime}$ the norm dual of $E$, and by $E_{+}^{\prime}$ the cone of all positive, linear, and continuous functionals on $E$. (See $[\mathrm{Ch}]$.)

Let $\psi: E \rightarrow \mathbb{R}$ be a concave and continuous functional. The directional derivative of $\psi$ at $x$ is

$$
d^{+} \psi(x)(v)=\lim _{t \rightarrow 0^{+}} \frac{\psi(x+t v)-\psi(x)}{t} \quad \forall v \in E .
$$

If the above limit exists for $t \rightarrow 0$ and all $v \in E, \psi$ is Gateaux differentiable at $x$, and the functional $\nabla \psi(x): v \mapsto d^{+} \psi(x)(v)$ is called Gateaux differential. (See $[\mathrm{Ph}]$ ). The superdifferential of $\psi$ at $x$ is the subset of $E^{\prime}$ defined by

$$
\partial \psi(x)=\left\{x^{\prime} \in E^{\prime}: \psi(y)-\psi(x) \leq\left\langle y-x, x^{\prime}\right\rangle \quad \forall y \in E\right\} ;
$$

while its Fenchel conjugate $\psi^{*}: E^{\prime} \rightarrow[-\infty, \infty)$ is given by

$$
\psi^{*}\left(x^{\prime}\right)=\inf _{x \in E}\left\{\left\langle x, x^{\prime}\right\rangle-\psi(x)\right\} \quad \forall x^{\prime} \in E^{\prime} .
$$

Finally, the domain of monotonicity of $\psi$ is the set $G_{\psi} \subseteq E$ given by

$$
G_{\psi}=\left\{x \in E: \partial \psi(x) \cap E_{+}^{\prime} \neq \varnothing\right\} .
$$

Next proposition confirms the intuition that the set $G_{\psi}$ is where the functional $\psi$ is monotone. 
Proposition 12 Let $E$ be an ordered Banach space and $\psi: E \rightarrow \mathbb{R}$ be a concave and continuous functional with $G_{\psi} \neq \varnothing$.

(i) The functional $\tilde{\psi}: E \rightarrow \mathbb{R}$, given by

$$
\tilde{\psi}(x)=\min _{x^{\prime} \in E_{+}^{\prime}}\left\{\left\langle x, x^{\prime}\right\rangle-\psi^{*}\left(x^{\prime}\right)\right\} \quad \forall x \in E,
$$

is the minimal monotone functional that dominates $\psi$; that is,

$$
\tilde{\psi}(x)=\sup \{\psi(y): y \in E \text { and } y \leqslant x\} \quad \forall x \in E .
$$

(ii) Let $x \in E, x \in G_{\psi} \Leftrightarrow d^{+} \psi(x)(v) \leq 0 \forall v \leqslant 0 \Leftrightarrow \tilde{\psi}(x)=\psi(x)$.

(iii) Let $x^{\prime} \in E^{\prime}$,

$$
(\tilde{\psi})^{*}\left(x^{\prime}\right)= \begin{cases}\psi^{*}\left(x^{\prime}\right) & \text { if } x^{\prime} \in E_{+}^{\prime} \\ -\infty & \text { otherwise }\end{cases}
$$

(iv) If $\psi^{*}$ is strictly concave on the intersection of its domain with $E_{+}^{\prime}$, then $\tilde{\psi}$ is Gateaux differentiable and $\nabla \tilde{\psi}(x)=\arg \min _{x^{\prime} \in E_{+}^{\prime}}\left\{\left\langle x, x^{\prime}\right\rangle-\psi^{*}\left(x^{\prime}\right)\right\}$ for all $x \in E$.

Moreover, if $G_{\psi}$ is convex, for all $x \in E$ there exists $y \in G_{\psi}$ such that $y \leqslant x$, and there exists a linear subspace $F \subseteq G_{\psi}$ such that $\psi_{\mid F}$ is linear and $\psi^{*}\left(x^{\prime}\right)$ is attained for all $x^{\prime} \in E_{+}^{\prime}$ with $x_{\mid F}^{\prime}=\psi_{\mid F}$; then, $\tilde{\psi}$ is the minimal monotone functional that extends $\psi_{\mid G_{\psi}}$ from $G_{\psi}$ to $E$. In this case,

$$
\tilde{\psi}(x)=\sup \left\{\psi(y): y \in G_{\psi} \text { and } y \leqslant x\right\}=\min _{x^{\prime} \in E_{+}^{\prime}: x_{\mid F}^{\prime}=\psi_{\mid F}}\left\{\left\langle x, x^{\prime}\right\rangle-\psi^{*}\left(x^{\prime}\right)\right\}
$$

for all $x \in E$.

By (i) and (ii), $\tilde{\psi}$ is the minimal monotone extension of $\psi_{\mid G_{\psi}}$ from $G_{\psi}$ to $E$ that dominates $\psi$ on $E$; in particular, $\tilde{\psi}(x) \geq \psi(x)$ for each $x \in E$ and $\tilde{\psi}(x)=\psi(x)$ for all $x \in G_{\psi}$. Moreover, (iii) shows that the Fenchel conjugates of $\tilde{\psi}$ and $\psi$ coincide on the cone $E_{+}^{\prime}$. (iv) is a useful differentiability property. The final part, shows that, under additional assumptions (satisfied, e.g., by the mean-variance functional), the extension $\tilde{\psi}$ has even stronger minimality properties. ${ }^{13}$

\footnotetext{
${ }^{13}$ Similar results can be obtained in greater generality, e.g., in the case of a proper concave, and upper semicontinuous function $\psi: E \rightarrow[-\infty, \infty)$ on a ordered locally convex space. Details are available upon request.
} 
Proof. By the Fenchel-Moreau Theorem, $\psi(x)=\inf _{x^{\prime} \in E^{\prime}}\left\{\left\langle x, x^{\prime}\right\rangle-\psi^{*}\left(x^{\prime}\right)\right\}$ for all $x \in E$. Set

$$
\tilde{\psi}(x)=\inf _{x^{\prime} \in E_{+}^{\prime}}\left\{\left\langle x, x^{\prime}\right\rangle-\psi^{*}\left(x^{\prime}\right)\right\} \quad \forall x \in E .
$$

Let $x_{0} \in G_{\psi}(\neq \varnothing)$ and $x_{0}^{\prime} \in \partial \psi\left(x_{0}\right) \cap E_{+}^{\prime}(\neq \varnothing)$, then

$$
\psi(x) \leq \psi\left(x_{0}\right)+\left\langle x, x_{0}^{\prime}\right\rangle-\left\langle x_{0}, x_{0}^{\prime}\right\rangle \quad \forall x \in E .
$$

Therefore, the functional $x_{0}^{\prime}+\left(\psi\left(x_{0}\right)-\left\langle x_{0}, x_{0}^{\prime}\right\rangle\right)$ is affine, monotone, and it dominates $\psi$. Notice that

$$
\left\langle x_{0}, x_{0}^{\prime}\right\rangle-\psi\left(x_{0}\right) \leq\left\langle x, x_{0}^{\prime}\right\rangle-\psi(x) \forall x \in E \text { and } \psi^{*}\left(x_{0}^{\prime}\right)=\left\langle x_{0}, x_{0}^{\prime}\right\rangle-\psi\left(x_{0}\right) .
$$

In particular, $x_{0}^{\prime} \in E_{+}^{\prime}$ and $\psi^{*}\left(x_{0}^{\prime}\right) \in \mathbb{R}$, hence, for all $x \in E$,

$$
-\infty<\psi(x) \leq \inf _{x^{\prime} \in E_{+}^{\prime}}\left\{\left\langle x, x^{\prime}\right\rangle-\psi^{*}\left(x^{\prime}\right)\right\}<\infty
$$

Then $\tilde{\psi}$ dominates $\psi$ and it takes finite values (i.e. it is a functional). Obviously, $\tilde{\psi}$ is concave and monotone.

(i) Let $\varphi: E \rightarrow \mathbb{R}$ be a concave and monotone functional such that $\varphi \geq \psi$. Then $\varphi$ is continuous, ${ }^{14}$ and $\varphi(x)=\inf _{x^{\prime} \in E_{+}^{\prime}}\left\{\left\langle x, x^{\prime}\right\rangle-\varphi^{*}\left(x^{\prime}\right)\right\}$ for all $x \in E .^{15}$ In addition, since $\varphi \geq \psi$, then $\varphi^{*} \leq \psi^{*}$ and

$\varphi(x)=\inf _{x^{\prime} \in E_{+}^{\prime}}\left\{\left\langle x, x^{\prime}\right\rangle-\varphi^{*}\left(x^{\prime}\right)\right\} \geq \inf _{x^{\prime} \in E_{+}^{\prime}}\left\{\left\langle x, x^{\prime}\right\rangle-\psi^{*}\left(x^{\prime}\right)\right\}=\tilde{\psi}(x) \quad \forall x \in E$.

This shows that $\tilde{\psi}$ is the minimal concave and monotone functional that dominates $\psi$. It is easy to check that the function defined by Eq. (11) is the minimal monotone functional that dominates $\psi$, and it is concave, hence it coincides with $\tilde{\psi}$.

(iii) Follows from the definition of $\tilde{\psi}$ (Eq. 14) and the observation that the function defined by Eq. (12) is proper, concave, and weak* upper semicontinuous.

\footnotetext{
${ }^{14}$ If $\varphi: \Omega \rightarrow \mathbb{R}$ is concave and monotone on an open subset $\Omega$ of an ordered Banach space, then it is continuous.

${ }^{15}$ If $\varphi: E \rightarrow[-\infty, \infty]$ is monotone and not identically $-\infty$, then $\varphi^{*}\left(x^{\prime}\right)=-\infty$ for all $x^{\prime} \notin E_{+}^{\prime}$.
} 
Since $\tilde{\psi}$ is concave and continuous, for all $x_{0} \in E,{ }^{16}$ (iii) delivers

$$
\begin{aligned}
x_{0}^{\prime} \in \partial \tilde{\psi}\left(x_{0}\right) & \Leftrightarrow x_{0}^{\prime} \in \arg \min _{x^{\prime} \in E^{\prime}}\left\{\left\langle x_{0}, x^{\prime}\right\rangle-\tilde{\psi}^{*}\left(x^{\prime}\right)\right\} \\
& \Leftrightarrow x_{0}^{\prime} \in \arg \min _{x^{\prime} \in E_{+}^{\prime}}\left\{\left\langle x_{0}, x^{\prime}\right\rangle-\psi^{*}\left(x^{\prime}\right)\right\} .
\end{aligned}
$$

This shows that the infimum in Eq. (14) is attained, and that (iv) holds.

(ii) If $x \in G_{\psi}$, then there is $x_{0}^{\prime} \in \partial \psi(x) \cap E_{+}^{\prime}$, and for all $v \leqslant 0$

$$
d^{+} \psi(x)(v)=\min _{x^{\prime} \in \partial \psi(x)}\left\langle v, x^{\prime}\right\rangle \leq\left\langle v, x_{0}^{\prime}\right\rangle \leq 0 .
$$

Conversely, assume that $d^{+} \psi(x)(v) \leq 0$ for all $v \leqslant 0$ and, by contradiction, that $x \notin G_{\psi}$, that is $\partial \psi(x) \cap E_{+}^{\prime}=\varnothing$. Since $E_{+}^{\prime}$ is a weak* closed and convex cone and $\partial \psi(x)$ is weak* compact, convex, and nonempty, there exists $v \in E$ such that

$$
\left\langle v, y^{\prime}\right\rangle \leq 0<\left\langle v, x^{\prime}\right\rangle \quad \forall y^{\prime} \in E_{+}^{\prime}, x^{\prime} \in \partial \psi(x) .
$$

Since $E_{+}$is closed, then $E_{+}=\left\{x \in E:\left\langle x, y^{\prime}\right\rangle \geq 0 \forall y^{\prime} \in E_{+}^{\prime}\right\}$. The left hand side of Eq. (16) amounts to say that $v \leqslant 0$, the right hand side that $\min _{x^{\prime} \in \partial \psi(x)}\left\langle v, x^{\prime}\right\rangle>0$, which is absurd.

If $x \in G_{\psi}$, then there is $x_{0}^{\prime} \in \partial \psi(x) \cap E_{+}^{\prime}$ that is

$$
\begin{aligned}
& x_{0}^{\prime} \in \arg \min _{x^{\prime} \in E^{\prime}}\left\{\left\langle x, x^{\prime}\right\rangle-\psi^{*}\left(x^{\prime}\right)\right\} \text { and } x_{0}^{\prime} \in E_{+}^{\prime}, \text { then } \\
& x_{0}^{\prime} \in \arg \min _{x^{\prime} \in E_{+}^{\prime}}\left\{\left\langle x, x^{\prime}\right\rangle-\psi^{*}\left(x^{\prime}\right)\right\} .
\end{aligned}
$$

The first line implies $\psi(x)=\left\langle x, x_{0}^{\prime}\right\rangle-\psi^{*}\left(x_{0}^{\prime}\right)$, the second that $\tilde{\psi}(x)=$ $\left\langle x, x_{0}^{\prime}\right\rangle-\psi^{*}\left(x_{0}^{\prime}\right)$. Conversely, if $\psi(x)=\min _{x^{\prime} \in E_{+}^{\prime}}\left\{\left\langle x, x^{\prime}\right\rangle-\psi^{*}\left(x^{\prime}\right)\right\}$, then there exists $x_{0}^{\prime} \in E_{+}^{\prime}$ such that

$$
\begin{aligned}
\left\langle x, x_{0}^{\prime}\right\rangle-\psi^{*}\left(x_{0}^{\prime}\right) & =\psi(x)=\min _{x^{\prime} \in E^{\prime}}\left\{\left\langle x, x^{\prime}\right\rangle-\psi^{*}\left(x^{\prime}\right)\right\}, \text { then } \\
x_{0}^{\prime} & \in \arg \min _{x^{\prime} \in E^{\prime}}\left\{\left\langle x, x^{\prime}\right\rangle-\psi^{*}\left(x^{\prime}\right)\right\} \text { and } x_{0}^{\prime} \in E_{+}^{\prime},
\end{aligned}
$$

therefore, $x_{0}^{\prime} \in \partial \psi(x) \cap E_{+}^{\prime}$ and $x \in G_{\psi}$.

Finally, assume $G_{\psi}$ is convex, for all $x \in E$ there exists $y \in G_{\psi}$ such that $y \leqslant x$, and there exists a linear subspace $F \subseteq G_{\psi}$ such that $\psi_{\mid F}$ is linear and $\psi^{*}\left(x^{\prime}\right)$ is attained for all $x^{\prime} \in E_{+}^{\prime}$ with $x_{\mid F}^{\prime}=\psi_{\mid F}$. Set

$$
\hat{\psi}(x)=\sup \left\{\psi(y): y \in G_{\psi} \text { and } y \leqslant x\right\} \quad \forall x \in E .
$$

\footnotetext{
${ }^{16}$ If $\varphi: E \rightarrow \mathbb{R}$ is concave and continuous, then, for all $x_{0} \in E$,$$
\partial \varphi\left(x_{0}\right)=\arg \min _{x^{\prime} \in E^{\prime}}\left\{\left\langle x_{0}, x^{\prime}\right\rangle-\varphi^{*}\left(x^{\prime}\right)\right\} \neq \varnothing .
$$ 
It is easy to check that $\hat{\psi}: E \rightarrow \mathbb{R}$ is the minimal monotone functional that extends $\psi_{\mid G_{\psi}}$ from $G_{\psi}$ to $E$, and that convexity of $G_{\psi}$ implies that it is concave. Therefore, $\hat{\psi} \leq \tilde{\psi}, \hat{\psi}(x)=\psi(x)$ for all $x \in G_{\psi}, \hat{\psi}$ is concave, monotone, and linear on $F$ (where it coincides with $\psi$ ). Hence, for all $x \in$ $E^{17}$

$$
\begin{aligned}
\hat{\psi}(x) & =\inf _{x^{\prime} \in E^{\prime}}\left\{\left\langle x, x^{\prime}\right\rangle-\hat{\psi}^{*}\left(x^{\prime}\right)\right\}=\inf _{x^{\prime} \in E_{+}^{\prime}: x_{\mid F}^{\prime}=\hat{\psi}_{\mid F}}\left\{\left\langle x, x^{\prime}\right\rangle-\hat{\psi}^{*}\left(x^{\prime}\right)\right\} \\
& =\inf _{x^{\prime} \in E_{+}^{\prime}: x_{\mid F}^{\prime}=\psi_{\mid F}}\left\{\left\langle x, x^{\prime}\right\rangle-\hat{\psi}^{*}\left(x^{\prime}\right)\right\} .
\end{aligned}
$$

For all $x^{\prime} \in E_{+}^{\prime}$ such that $x_{\mid F}^{\prime}=\psi_{\mid F}$,

$$
\hat{\psi}^{*}\left(x^{\prime}\right)=\inf _{x \in E}\left\{\left\langle x, x^{\prime}\right\rangle-\hat{\psi}(x)\right\} \leq \inf _{x \in G_{\psi}}\left\{\left\langle x, x^{\prime}\right\rangle-\hat{\psi}(x)\right\}=\inf _{x \in G_{\psi}}\left\{\left\langle x, x^{\prime}\right\rangle-\psi(x)\right\} .
$$

Analogously, since $\psi$ is linear on $F$ then, for all $x \in E$,

$$
\tilde{\psi}(x)=\min _{x^{\prime} \in E_{+}^{\prime}: x_{\mid F}^{\prime}=\psi_{\mid F}}\left\{\left\langle x, x^{\prime}\right\rangle-\psi^{*}\left(x^{\prime}\right)\right\} .
$$

Since $\psi^{*}\left(x^{\prime}\right)$ is attained for all $x^{\prime} \in E_{+}^{\prime}$ such that $x_{\mid F}^{\prime}=\psi_{\mid F}$, for every $x^{\prime}$ with these properties there exists $x_{0} \in E$ such that

$$
\left\langle x_{0}, x^{\prime}\right\rangle-\psi\left(x_{0}\right) \leq\left\langle x, x^{\prime}\right\rangle-\psi(x) \quad \forall x \in E .
$$

Then $x^{\prime} \in \partial \psi\left(x_{0}\right) \cap E_{+}^{\prime}$ and $x_{0} \in G_{\psi}$, in particular, $\psi^{*}\left(x^{\prime}\right)$ is attained in $G_{\psi}$. That is, for $x^{\prime} \in E_{+}^{\prime}$ such that $x_{\mid F}^{\prime}=\psi_{\mid F}$,

$$
\psi^{*}\left(x^{\prime}\right)=\inf _{x \in G_{\psi}}\left\{\left\langle x, x^{\prime}\right\rangle-\psi(x)\right\} \geq \hat{\psi}^{*}\left(x^{\prime}\right)
$$

where the last inequality descends from Eq. (17). We conclude that $\hat{\psi}(x)=\inf _{x^{\prime} \in E_{+}^{\prime}: x_{\mid F}^{\prime}=\psi_{\mid F}}\left\{\left\langle x, x^{\prime}\right\rangle-\hat{\psi}^{*}\left(x^{\prime}\right)\right\} \geq \inf _{x^{\prime} \in E_{+}^{\prime}: x_{\mid F}^{\prime}=\psi_{\mid F}}\left\{\left\langle x, x^{\prime}\right\rangle-\psi^{*}\left(x^{\prime}\right)\right\}=\tilde{\psi}(x)$ for all $x \in E$, as wanted.

These results have been recently extended in Filipovic and Kupper [FK-2].

\footnotetext{
${ }^{17}$ If $\varphi: E \rightarrow[-\infty, \infty]$ is linear on a subspace $F$, then $\varphi^{*}\left(x^{\prime}\right)=-\infty$ if $x_{\mid F}^{\prime} \neq \varphi_{\mid F}$.
} 


\section{B Proofs}

Let $f \in \mathcal{L}(P)$, we denote by $F_{f}(t)=P(f \leq t)$ its cumulative distribution function and by $g_{f}(t)=\int_{-\infty}^{t} F_{f}(z) d z$ its integral distribution function.

Next two lemmas regroup some useful properties of integrated distribution functions. We report the proofs for the sake of completeness.

Lemma 13 For all $z \in \mathbb{R}$,

$$
\begin{array}{r}
g_{f}(z)=\int(f-z)^{-} d P \\
=z P(f \leq z)-\int f 1_{\{f \leq z\}} d P \\
=z P(f<z)-\int f 1_{\{f<z\}} d P \\
=\int z-(f \wedge z) d P \\
=\int_{-\infty}^{z} P(f<t) d t .
\end{array}
$$

Proof. Let $z \in \mathbb{R} .(f-z)^{-}=(z-f) 1_{\{f \leq z\}}$, hence

$$
\int(f-z)^{-} d P=z P(f \leq z)-\int f 1_{\{f \leq z\}} d P .
$$

Moreover,

$$
\begin{aligned}
z P(f \leq z)-\int f 1_{\{f \leq z\}} d P & =z P(f<z)+z P(f=z)-\int_{\{f<z\}} f d P-\int_{\{f=z\}} f d P \\
& =z P(f<z)+z P(f=z)-\int_{\{f<z\}} f d P-z P(f=z) \\
& =z P(f<z)-\int f 1_{\{f<z\}} d P .
\end{aligned}
$$

Since $f 1_{\{f \leq z\}}=(f \wedge z)-z 1_{\{f>z\}}$, then

$$
\begin{aligned}
z P(f \leq z)-\int f 1_{\{f \leq z\}} d P & =z P(f \leq z)-\int\left(f \wedge z-z 1_{\{f>z\}}\right) d P \\
& =z P(f \leq z)-\int(f \wedge z) d P+z \int 1_{\{f>z\}} d P \\
& =z P(f \leq z)+z P(f>z)-\int(f \wedge z) d P \\
& =\int z-(f \wedge z) d P .
\end{aligned}
$$


Observe that $z-(f \wedge z) \geq 0$, and so

$$
\int z-(f \wedge z) d P=\int_{0}^{\infty} P(z-(f \wedge z) \geq u) d u
$$

On the other hand, $\{z-(f \wedge z) \geq u\}=\{f \leq z-u\}$ for all $u>0$. In fact,

$$
z-(f \wedge z) \geq u \Rightarrow(f \wedge z) \leq z-u<z \Rightarrow(f \wedge z)=f \Rightarrow f \leq z-u
$$

and

$$
f \leq z-u \Rightarrow f \wedge z \leq(z-u) \wedge z \Rightarrow f \wedge z \leq z-u \Rightarrow z-(f \wedge z) \geq u .
$$

Hence,

$$
\begin{aligned}
\int z-(f \wedge z) d P & =\int_{0}^{\infty} P(z-(f \wedge z) \geq u) d u \\
& =\int_{0}^{\infty} P(f \leq z-u) d u=\int_{-\infty}^{z} P(f \leq t) d t=g_{f}(z),
\end{aligned}
$$

thus the first four equalities hold.

Finally, notice that $P(f<t)=\lim _{u \rightarrow t^{-}} P(f \leq u) \neq P(f \leq t)$ for at most a countably many $t$ s.

Lemma 14 The function $g_{f}: \mathbb{R} \rightarrow[0, \infty)$ is continuous and

$$
F_{f}(z)=\lim _{\varepsilon \rightarrow 0^{+}}\left[\frac{g_{f}(z+\varepsilon)-g_{f}(z)}{\varepsilon}\right] \quad \forall z \in \mathbb{R} .
$$

That is, $F_{f}$ is the right derivative of $g_{f}$, and $F_{f}(z)$ is the derivative of $g_{f}$ at every point $z$ at which $F_{f}$ is continuous. Moreover, setting $\zeta=\operatorname{essinf}(f), g_{f}$ is strictly increasing on $(\zeta, \infty), g_{f} \equiv 0$ on $(-\infty, \zeta],{ }^{18} \lim _{z \rightarrow \zeta^{+}} g_{f}(z)=0^{+}$, and $\lim _{z \rightarrow \infty} g_{f}(z)=\infty$.

Proof. The Fundamental Theorem of Calculus guarantees the continuity and derivability properties of $g_{f}$. Recall that

$$
\operatorname{essinf}(f)=\sup \{\alpha \in \mathbb{R}: P(f<\alpha)=0\} .
$$

If $z \in \mathbb{R}$ and $z \leq \operatorname{essinf}(f)$, for all $t<z$ there exists $\alpha>t$ such that $P(f<\alpha)=0$. Then,

$$
0 \leq P(f<t) \leq P(f<\alpha)=0 .
$$

\footnotetext{
${ }^{18}$ With the convention $(-\infty,-\infty]=\varnothing$.
} 
This implies $g_{f}(z)=0$ for all $z \in(-\infty, \zeta]$.

On the other hand, if $\zeta<z<z^{\prime}$, then

$$
g_{f}\left(z^{\prime}\right)-g_{f}(z)=\int_{z}^{z^{\prime}} P(f<t) d t \geq P(f<z)\left(z^{\prime}-z\right) .
$$

But $P(f<z)=0$ would imply $z \leq \zeta$, a contradiction. Therefore, $g_{f}\left(z^{\prime}\right)-$ $g_{f}(z)>0$. That is, $g_{f}$ is strictly increasing on $(\zeta, \infty)$.

Notice that $\lim _{t \rightarrow \infty} F_{f}(t)=1$. Then, for all $n>1$ there exists $k \geq 1$ $(n, k \in \mathbb{N})$ such that $F_{f}(t)>1-\frac{1}{n}$ for all $t \geq k$. Therefore,

$$
\begin{aligned}
g_{f}(k+n) & =\int_{-\infty}^{k+n} F_{f}(t) d t \geq \int_{k}^{k+n} F_{f}(t) d t \\
& \geq n\left(1-\frac{1}{n}\right)=n-1 .
\end{aligned}
$$

Since $g_{f}$ is increasing on $\mathbb{R}$, then $\lim _{z \rightarrow \infty} g_{f}(z)=\infty$.

If $\zeta>-\infty, g_{f}(\zeta)=0$, continuity and nonnegativity imply $\lim _{z \rightarrow \zeta^{+}} g_{f}(z)=$ $0^{+}$. Let $\zeta=-\infty$, for all $n>1$,

$$
\begin{aligned}
g_{f}(-n) & =\int(-n-(f \wedge(-n))) d P=\int(((-f) \vee n)-n) d P \\
& =\int((-f)-((-f) \wedge n)) d P .
\end{aligned}
$$

The Monotone Convergence Theorem guarantees that $\lim _{n \rightarrow \infty} g_{f}(-n)=0$. Monotonicity and nonnegativity imply $\lim _{z \rightarrow \zeta^{+}} g_{f}(z)=0^{+}$.

For the rest of the Appendix we indifferently write $\mathrm{E}^{P}$ or just E. Denoting by $\geqslant$ the relation $\geq P$-a.s., $\left(\mathcal{L}^{2}(P),\|\cdot\|_{2}, \geqslant\right)$ is an ordered Banach space, and its norm dual can be identified with $\mathcal{L}^{2}(P)$, with the duality relation $\langle f, Y\rangle=\mathrm{E}[f Y]$. Simple computation shows that, for all $\theta>0$,

$$
\left\{f \in \mathcal{L}^{2}(P): \nabla U_{\theta}(f) \geqslant 0\right\}=\left\{f \in \mathcal{L}^{2}(P): f-\mathrm{E}^{P}[f] \leqslant \frac{1}{\theta}\right\}
$$

this set is denoted by $\mathcal{G}_{\theta}$.

Lemma 15 Let $f \in \mathcal{L}^{2}(P)-\mathcal{G}_{\theta}$ and $t \in \mathbb{R}$. Then

$$
f \wedge t \in \mathcal{G}_{\theta} \Leftrightarrow g_{f}(t) \leq \frac{1}{\theta}
$$


Proof. Notice that

$$
\begin{aligned}
f \wedge t-\mathrm{E}[f \wedge t] & =f 1_{\{f \leq t\}}+t 1_{\{f>t\}}-t P(f>t)-\mathrm{E}\left[f 1_{\{f \leq t\}}\right] \\
& =f 1_{\{f \leq t\}}+t 1_{\{f>t\}}-t+t P(f \leq t)-\mathrm{E}\left[f 1_{\{f \leq t\}}\right] \\
& =(f-t) 1_{\{f \leq t\}}+g_{f}(t) .
\end{aligned}
$$

Since $(f-t) 1_{\{f \leq t\}} \leqslant 0$,

$$
g_{f}(t) \leq \frac{1}{\theta} \Rightarrow f \wedge t-\mathrm{E}[f \wedge t] \leqslant \frac{1}{\theta}
$$

i.e., $g_{f}(t) \leq \frac{1}{\theta} \Rightarrow f \wedge t \in \mathcal{G}_{\theta}$.

For the converse implication, notice that we are assuming $f \notin \mathcal{G}_{\theta}$. Then, being $f \wedge t \in \mathcal{G}_{\theta}$, it cannot be $f \wedge t=f P$-a.s.. Hence, essup $(f \wedge t)=t$. It follows that:

$$
\begin{aligned}
f \wedge t-\mathrm{E}[f \wedge t] \leqslant \frac{1}{\theta} & \Rightarrow \operatorname{essup}(f \wedge t)-t P(f>t)-\mathrm{E}\left[f 1_{\{f \leq t\}}\right] \leq \frac{1}{\theta} \\
& \Rightarrow t-t P(f>t)-\mathrm{E}\left[f 1_{\{f \leq t\}}\right] \leq \frac{1}{\theta} \\
& \Rightarrow t P(f \leq t)-\mathrm{E}\left[f 1_{\{f \leq t\}}\right] \leq \frac{1}{\theta} \\
& \Rightarrow g_{f}(t) \leq \frac{1}{\theta},
\end{aligned}
$$

i.e., $f \wedge t \in \mathcal{G}_{\theta} \Rightarrow g_{f}(t) \leq \frac{1}{\theta}$.

Lemmas 14 and 15 immediately yield the following:

Corollary 16 Let $f \in \mathcal{L}^{2}(P)-\mathcal{G}_{\theta}$, then $g_{f}^{-1}\left(\frac{1}{\theta}\right)=\max \left\{t \in \mathbb{R}: f \wedge t \in \mathcal{G}_{\theta}\right\}$.

Lemma 17 For all $\theta>0$,

(i) $\mathcal{G}_{\theta}$ is convex, closed, and for all $f \in \mathcal{L}^{2}(P)-\mathcal{G}_{\theta}$ and every $\varepsilon>0$ there exists $g \in \mathcal{L}^{2}(P)$ such that $|f(s)-g(s)|<\varepsilon$ for all $s \in S, g>f$, and $U_{\theta}(g)<U_{\theta}(f)$.

(ii) $U_{\theta}$ is linear on the subspace $\mathcal{T} \subseteq \mathcal{G}_{\theta}$ of all P-a.s. constant functions.

(iii) For all $Y \in \mathcal{L}^{2}(P)$,

$$
U_{\theta}^{*}(Y)= \begin{cases}-\frac{1}{2 \theta}\left(\mathrm{E}\left[Y^{2}\right]-1\right) & \text { if } \mathrm{E}[Y]=1, \\ -\infty & \text { otherwise. }\end{cases}
$$

(iv) $\left\{Y \in \mathcal{L}^{2}(P): \mathrm{E}[Y]=1\right\}=\left\{Y \in \mathcal{L}^{2}(P):\langle\cdot, Y\rangle_{\mid \mathcal{T}}=U_{\theta \mid \mathcal{T}}\right\}$ and $U_{\theta}^{*}$ is attained and strictly concave on this set. 
Proof. (i) Convexity of $\mathcal{G}_{\theta}$ is trivial. Next we show closure. If $f_{n} \in \mathcal{G}_{\theta}$ and $f_{n} \rightarrow f$ in $\mathcal{L}^{2}(P)$, then there exists a subsequence $g_{n}$ of $f_{n}$ such that $g_{n}(s) \rightarrow f(s)$ for $P$-almost all $s$ in $S$. Let $A_{0}=\left\{s \in S: g_{n}(s) \rightarrow f(s)\right\}$, and for all $n \geq 1, A_{n}=\left\{s \in S: g_{n}(s)-\mathrm{E}\left[g_{n}\right] \leq 1 / \theta\right\}$, then $P\left(\bigcap_{n \geq 0} A_{n}\right)=1$ and for all $s \in \bigcap_{n \geq 0} A_{n}$,

$$
f(s)-\mathrm{E}[f]=\lim _{n \rightarrow \infty}\left(g_{n}(s)-\mathrm{E}\left[g_{n}\right]\right) \leq \frac{1}{\theta}
$$

that is $f-\mathrm{E}[f] \leqslant 1 / \theta$.

Moreover, if $f \notin \mathcal{G}_{\theta}$, then $\nabla U_{\theta}(f)$ is not positive and there exists $A$ with $P(A)>0$ such that $\int 1_{A} \nabla U_{\theta}(f) d P<0$, then

$$
\lim _{t \rightarrow 0} \frac{U_{\theta}\left(f+t 1_{A}\right)-U_{\theta}(f)}{t}=\int 1_{A} \nabla U_{\theta}(f) d P<0
$$

that is, there exists $\varepsilon>0$ such that

$$
\frac{U_{\theta}\left(f+t 1_{A}\right)-U_{\theta}(f)}{t}<0
$$

for all $t \in(0, \varepsilon)$. As a consequence for all such $t \mathrm{~s}, f+t 1_{A}>f, U_{\theta}\left(f+t 1_{A}\right)<$ $U_{\theta}(f)\left(\operatorname{set} g=f+(\varepsilon / 2) 1_{A}\right)$.

(ii) is trivial.

For convenience, (iii) and (iv) are proved together. For all $t \in \mathbb{R}, U_{\theta}\left(t 1_{S}\right)=$ $t$, then for all $Y \in \mathcal{L}^{2}(P)$

$$
\left\{Y \in \mathcal{L}^{2}(P):\langle\cdot, Y\rangle_{\mid \mathcal{T}}=U_{\theta \mid \mathcal{T}}\right\}=\left\{Y \in \mathcal{L}^{2}(P): \mathrm{E}[Y]=1\right\}
$$

and $U_{\theta}^{*}(Y)=-\infty$ for all $Y$ that does not belong to this set.

If $\mathrm{E}[Y]=1$, the functional $W_{\theta}: \mathcal{L}^{2}(P) \rightarrow \mathbb{R}$ given, for all $f \in \mathcal{L}^{2}(P)$, by

$$
W_{\theta}(f)=\langle f, Y\rangle-U_{\theta}(f)=\langle f, Y\rangle-\langle f, 1\rangle+\frac{\theta}{2}\langle f-\mathrm{E}[f], f-\mathrm{E}[f]\rangle
$$

is well defined, convex, and Gateaux differentiable. Its Gateaux differential is

$$
\nabla W_{\theta}(f)=Y-1+\theta(f-\mathrm{E}[f]) .
$$

Notice that $\hat{f}=-\theta^{-1} Y$ solves $\nabla W_{\theta}(\hat{f})=0$ (since $\mathrm{E}[Y]=1$ ), and $W_{\theta}$ attains its minimum on $\mathcal{L}^{2}(P)$ at $\hat{f}$. This implies that $U_{\theta}^{*}(Y)=\inf _{f \in \mathcal{L}^{2}(P)} W_{\theta}(f)$ is attained, and

$$
\begin{aligned}
U_{\theta}^{*}(Y) & =\min _{f \in \mathcal{L}^{2}(P)} W_{\theta}(f)=W_{\theta}(\hat{f})=\left\langle-\frac{1}{\theta} Y, Y\right\rangle-\left\langle-\frac{1}{\theta} Y, 1\right\rangle+\frac{\theta}{2} \operatorname{Var}\left[-\frac{1}{\theta} Y\right] \\
& =-\frac{1}{\theta} \mathrm{E}\left[Y^{2}\right]+\frac{1}{\theta} \mathrm{E}[Y]+\frac{\theta}{2} \frac{1}{\theta^{2}} \operatorname{Var}[Y]=-\frac{1}{2 \theta}\left(\mathrm{E}\left[Y^{2}\right]-1\right) .
\end{aligned}
$$


This concludes the proof of Eq. (21). Strict concavity of $U_{\theta}^{*}$ in its domain is a straightforward application of the the Cauchy-Schwartz inequality.

Proof of Lemma 1. It is an immediate consequence of Eq. (19) and part (i) of the above Lemma 17 .

Proof of Theorem 2. As observed, $U_{\theta}: \mathcal{L}^{2}(P) \rightarrow \mathbb{R}$ is a concave and continuous functional on an ordered Banach space, $\mathcal{G}_{\theta}=G_{U_{\theta}}$, Lemma 17 and Corollary 16, guarantee that all the hypotheses of Proposition 12 are satisfied. For all $f \in \mathcal{L}^{2}(P)$,

$$
\begin{aligned}
\tilde{U}_{\theta}(f) & =\min _{Y \in \mathcal{L}_{+}^{2}(P): \mathrm{E}[Y]=1}\left\{\mathrm{E}[f Y]+\frac{1}{2 \theta}\left(\mathrm{E}\left[Y^{2}\right]-1\right)\right\} \\
& =\min _{Q \in \Delta^{2}(P)}\left\{\mathrm{E}^{Q}[f]+\frac{1}{2 \theta} C(Q \| P)\right\}=V_{\theta}(f),
\end{aligned}
$$

and $V_{\theta}$ has all the desired properties.

Remark 18 Proposition 12.(iv) and Lemma 17.(iv) guarantee that $V_{\theta}$ is Gateaux differentiable, and

$$
\nabla V_{\theta}(f)=\arg \min _{Y \in \mathcal{L}_{+}^{2}(P): \mathrm{E}[Y]=1}\left\{\mathrm{E}[f Y]+\frac{1}{2 \theta}\left(\mathrm{E}\left[Y^{2}\right]-1\right)\right\} \quad \forall f \in \mathcal{L}^{2}(P) .
$$

Theorem 19 Let $f \in \mathcal{L}^{2}(P)$. Then

$$
V_{\theta}(f)= \begin{cases}\mathrm{E}[f]-\frac{\theta}{2} \operatorname{Var}[f] & \text { if } f \in \mathcal{G}_{\theta} \\ \mathrm{E}[f \wedge \kappa]-\frac{\theta}{2} \operatorname{Var}[f \wedge \kappa] & \text { else }\end{cases}
$$

where $\kappa=g_{f}^{-1}\left(\frac{1}{\theta}\right)$. Moreover, the Gateaux differential of $V_{\theta}$ at $f$ is

$$
\nabla V_{\theta}(f)=\theta(\kappa-f) 1_{\{f \leq \kappa\}} .
$$

Proof. For all $f \in \mathcal{L}^{2}(P), V_{\theta}(f)=\min _{Q \in \Delta^{2}(P)}\left\{\mathrm{E}^{Q}(f)+\frac{1}{2 \theta} C(Q \| P)\right\}$. That is, $V_{\theta}(f)$ is the value of the problem:

$$
\left\{\begin{array}{l}
\min \left\{\mathrm{E}[f Y]+\frac{1}{2 \theta} \mathrm{E}\left[Y^{2}\right]-\frac{1}{2 \theta}\right\} \\
Y \geqslant 0 \\
E[Y]=1
\end{array} .\right.
$$

Remark 18 guarantees that the solution of such problem exists, is unique, and it coincides with the Gateaux derivative of $V_{\theta}$ at $f$. Notice that $Y$ is 
a solution of problem (24) if and only if it is a solution of the constrained optimization problem:

$$
\left\{\begin{array}{l}
\min \left\{\mathrm{E}[f Y]+\frac{1}{2 \theta} \mathrm{E}\left[Y^{2}\right]\right\} \\
Y \geqslant 0 \\
\mathrm{E}[Y]=1
\end{array} .\right.
$$

The Lagrangian is

$$
L(Y, \mu, \lambda)=\mathrm{E}(f Y)+\frac{1}{2 \theta} \mathrm{E}\left(Y^{2}\right)-\mathrm{E}(\mu Y)-\lambda(\mathrm{E}(Y)-1),
$$

with $\mu \in \mathcal{L}_{+}^{2}(P), \lambda \in \mathbb{R}$. The Kuhn-Tucker optimality conditions are:

$$
\begin{aligned}
& f+\frac{1}{\theta} Y-\mu-\lambda=0 \quad(P \text {-a.s. }) \\
& \mathrm{E}(\mu Y)=0 \\
& Y \geqslant 0, \mu \geqslant 0 \\
& \mathrm{E}(Y)=1
\end{aligned}
$$

Since $\mu, Y \geqslant 0$, they are equivalent to:

$$
\begin{aligned}
& f+\frac{1}{\theta} Y-\mu-\lambda=0 \quad(P \text {-a.s. }) \\
& \mu Y=0 \quad(P \text {-a.s. }) \\
& Y \geqslant 0, \mu \geqslant 0 \\
& \mathrm{E}(Y)=1
\end{aligned}
$$

that is,

$$
\begin{gathered}
f+\frac{1}{\theta} Y-\lambda \geq 0 \quad P \text {-a.s. } \\
{\left[f+\frac{1}{\theta} Y-\lambda\right] Y=0 \quad P \text {-a.s. }} \\
Y \geq 0 \quad P \text {-a.s. } \\
\mathrm{E}[Y]=1
\end{gathered}
$$

It is sufficient to find $\left(Y^{*}, \lambda^{*}\right)$ that satisfy $(26)$ - (29) everywhere (not only $P$-a.s. $)$.

If $s \in\left\{Y^{*}>0\right\}$, then by (27) $f(s)+\frac{1}{\theta} Y^{*}(s)-\lambda^{*}=0$ and

$$
Y^{*}(s)=\theta\left(\lambda^{*}-f(s)\right) .
$$


In particular, $\lambda^{*}-f(s)>0$, and $s \in\left\{f<\lambda^{*}\right\}$. Conversely, if $s \in\left\{f<\lambda^{*}\right\}$, then by (26) $Y^{*}(s) \geq \theta\left(\lambda^{*}-f(s)\right)>0$ and $s \in\left\{Y^{*}>0\right\}$. In sum,

$$
\begin{aligned}
& \left\{Y^{*}>0\right\}=\left\{f<\lambda^{*}\right\} \text { and } \\
& Y^{*}=\theta\left(\lambda^{*}-f\right) 1_{\left\{f<\lambda^{*}\right\}} .
\end{aligned}
$$

By (29),

$$
\begin{aligned}
1 & =\mathrm{E}\left[Y^{*}\right]=\mathrm{E}\left[\theta\left(\lambda^{*}-f\right) 1_{\left\{f<\lambda^{*}\right\}}\right] \\
& =\theta\left(\lambda^{*} P\left(f<\lambda^{*}\right)-\mathrm{E}\left[f 1_{\left\{f<\lambda^{*}\right\}}\right]\right),
\end{aligned}
$$

that is,

$$
g_{f}\left(\lambda^{*}\right)=\lambda^{*} P\left(f<\lambda^{*}\right)-\mathrm{E}\left[f 1_{\left\{f<\lambda^{*}\right\}}\right]=\frac{1}{\theta} .
$$

In other words,

$$
\lambda^{*}=g_{f}^{-1}\left(\frac{1}{\theta}\right) \equiv \kappa
$$

and $\lambda^{*}$ is unique. A fortiori, $Y^{*}$ is unique and

$$
Y^{*}=\theta(\kappa-f) 1_{\{f<\kappa\}} .
$$

By construction, the pair $\left(Y^{*}, \lambda^{*}\right)$ defined by (31) and (32) is a solution of (26) - (29). Since the solution of (24) exists and it is unique, we conclude that $Y^{*}$ defined as in Eq. (32) is the unique solution of (24).

Notice that $Y^{*}=\theta(\kappa-f) 1_{\{f<\kappa\}}+\theta(\kappa-f) 1_{\{f=\kappa\}}=\theta(\kappa-f) 1_{\{f \leq \kappa\}}$,

$$
\left(Y^{*}\right)^{2}=\theta^{2}\left(f^{2} 1_{\{f \leq \kappa\}}+\kappa^{2} 1_{\{f \leq \kappa\}}-2 \kappa f 1_{\{f \leq \kappa\}}\right)
$$

and

$$
\mathrm{E}\left[\left(Y^{*}\right)^{2}\right]=\theta^{2}\left(\int f^{2} 1_{\{f \leq \kappa\}} d P+\kappa^{2} P(f \leq \kappa)-2 \kappa \int f 1_{\{f \leq \kappa\}} d P\right) .
$$

Moreover,

$$
\begin{aligned}
\mathrm{E}\left[f Y^{*}\right] & =\mathrm{E}\left[f \theta(\kappa-f) 1_{\{f \leq \kappa\}}\right]=\mathrm{E}\left[\theta \kappa f 1_{\{f \leq \kappa\}}-\theta f^{2} 1_{\{f \leq \kappa\}}\right] \\
& =\theta \kappa \int f 1_{\{f \leq \kappa\}} d P-\theta \int f^{2} 1_{\{f \leq \kappa\}} d P .
\end{aligned}
$$


Therefore,

$$
\begin{aligned}
V_{\theta}(f) & =\mathrm{E}\left[f Y^{*}\right]+\frac{1}{2 \theta} \mathrm{E}\left[\left(Y^{*}\right)^{2}\right]-\frac{1}{2 \theta} \\
& =\theta \kappa \int f 1_{\{f \leq \kappa\}} d P-\theta \int f^{2} 1_{\{f \leq \kappa\}} d P+ \\
& +\frac{\theta}{2}\left(\int f^{2} 1_{\{f \leq \kappa\}} d P+\kappa^{2} P(f \leq k)-2 \kappa \int f 1_{\{f \leq \kappa\}} d P\right)-\frac{1}{2 \theta} \\
& =-\frac{\theta}{2} \int f^{2} 1_{\{f \leq \kappa\}} d P+\frac{\theta}{2} \kappa^{2} P(f \leq k)-\frac{1}{2 \theta}
\end{aligned}
$$

Also observe that $f 1_{\{f \leq \kappa\}}+\kappa 1_{\{f>\kappa\}}=f \wedge \kappa$, whence

$$
\begin{aligned}
\mathrm{E}[f \wedge \kappa] & =\mathrm{E}\left[f 1_{\{f \leq \kappa\}}\right]+\kappa P(f>\kappa)=\mathrm{E}\left[f 1_{\{f \leq \kappa\}}\right]-\kappa P(f \leq \kappa)+\kappa \\
& =-g_{f}(\kappa)+\kappa=\kappa-\frac{1}{\theta},
\end{aligned}
$$

and

$$
\begin{aligned}
\operatorname{Var}[f \wedge \kappa] & =\mathrm{E}\left[\left(f 1_{\{f \leq \kappa\}}+\kappa 1_{\{f>\kappa\}}\right)^{2}\right]-\left(\kappa-\frac{1}{\theta}\right)^{2} \\
& =\int f^{2} 1_{\{f \leq \kappa\}} d P+\kappa^{2} P(f>k)-\kappa^{2}-\frac{1}{\theta^{2}}+2 \frac{\kappa}{\theta} \\
& =\int f^{2} 1_{\{f \leq \kappa\}} d P-\kappa^{2} P(f \leq k)-\frac{1}{\theta^{2}}+2 \frac{\kappa}{\theta}
\end{aligned}
$$

Finally,

$$
\begin{aligned}
\mathrm{E}[f \wedge \kappa]-\frac{\theta}{2} \operatorname{Var}[f \wedge \kappa] & =\kappa-\frac{1}{\theta}-\frac{\theta}{2}\left(\int f^{2} 1_{\{f \leq \kappa\}} d P-\kappa^{2} P(f \leq k)-\frac{1}{\theta^{2}}+2 \frac{\kappa}{\theta}\right) \\
& =\kappa-\frac{1}{\theta}-\left(\frac{\theta}{2} \int f^{2} 1_{\{f \leq \kappa\}} d P-\frac{\theta}{2} \kappa^{2} P(f \leq k)-\frac{1}{2 \theta}+\kappa\right) \\
& =-\frac{\theta}{2} \int f^{2} 1_{\{f \leq \kappa\}} d P+\frac{\theta}{2} \kappa^{2} P(f \leq k)-\frac{1}{2 \theta} \\
& =V_{\theta}(f) .
\end{aligned}
$$

Proof of Theorem 3. It is now enough to combine Corollary 16 and Theorem 19.

Remark 20 Inspection of the proof of Theorem 19 shows that: 
- For all $f \in \mathcal{L}^{2}(P)$ (not only for $f \in \mathcal{L}^{2}(P)-\mathcal{G}_{\theta}$ ), setting $\kappa=g_{f}^{-1}\left(\frac{1}{\theta}\right)$ we have

$$
\begin{aligned}
V_{\theta}(f) & =\mathrm{E}[f \wedge \kappa]-\frac{\theta}{2} \operatorname{Var}[f \wedge \kappa], \\
\nabla V_{\theta}(f) & =\theta(\kappa-f) 1_{\{f \leq \kappa\}}=\theta(f-\kappa)^{-}, \\
\nabla V_{\theta}(f) & =\arg \min _{Q \in \Delta^{2}(P)}\left\{\mathrm{E}^{Q}(f)+\frac{1}{2 \theta} C(Q \| P)\right\} .
\end{aligned}
$$

- The properties of $g_{f}$ guarantee that $\kappa$ exists, it is unique, and $\frac{1}{\theta}=$ $\kappa P(f \leq \kappa)-\int f 1_{\{f \leq \kappa\}} d P$; therefore, $P(f \leq \kappa)>0$.

- Moreover, $\frac{1}{\theta}=\kappa P(f \leq \kappa)-\int f 1_{\{f \leq \kappa\}} d P$ implies $\frac{1}{\theta P(f \leq \kappa)}+\int f d P_{\{f \leq \kappa\}}=$ $\kappa$, and so

$$
\begin{aligned}
\nabla V_{\theta}(f) & =\theta(\kappa-f) 1_{\{f \leq \kappa\}}=\left(\frac{1}{P(f \leq \kappa)}+\theta \int f d P_{\{f \leq \kappa\}}-\theta f\right) 1_{\{f \leq \kappa\}} \\
& =\left(\frac{1}{P(f \leq \kappa)}-\theta(f-\mathrm{E}[f \mid f \leq \kappa])\right) 1_{\{f \leq \kappa\}} .
\end{aligned}
$$

Proof of Theorem 5. By Theorem 13.8 of Chong and Rice [CR], for all $f, Y \in \mathcal{L}^{2}(P)$, the set $\left\{\int f Y^{\prime} d P: Y^{\prime} \in \mathcal{L}^{2}(P)\right.$ and $\left.Y^{\prime} \succeq_{c} Y\right\}$ coincides with the interval

$$
\left[\int_{0}^{1} F_{f}^{-1}(1-u) F_{Y}^{-1}(u) d u, \int_{0}^{1} F_{f}^{-1}(u) F_{Y}^{-1}(u) d u\right]
$$

where $\succeq_{c}$ is the concave order and $F_{f}^{-1}$ is the quantile function of $f \cdot{ }^{19}$ Consider the function $\Gamma: \mathcal{L}^{2}(P) \rightarrow[0, \infty]$ defined by

$$
\Gamma(Y)= \begin{cases}\frac{1}{2 \theta}\left(\mathrm{E}\left[Y^{2}\right]-1\right) & \text { if } Y \geqslant 0 \text { and } \mathrm{E}[Y]=1 \\ \infty & \text { otherwise. }\end{cases}
$$

It is easy to check that $Y^{\prime} \succeq_{c} Y$ implies $\Gamma\left(Y^{\prime}\right) \leq \Gamma(Y) .{ }^{20}$ Let $f \in \mathcal{L}^{2}(P)$,

$V_{\theta}(f)=\min _{Y \in \mathcal{L}^{2}(P)}\{\mathrm{E}[f Y]+\Gamma(Y)\} \geq \inf _{Y \in \mathcal{L}^{2}(P)}\left\{\int_{0}^{1} F_{f}^{-1}(1-u) F_{Y}^{-1}(u) d u+\Gamma(Y)\right\}$.

\footnotetext{
${ }^{19}$ I.e. $\quad Y^{\prime} \succeq_{c} Y$ iff $\int \phi\left(Y^{\prime}\right) d P \geq \int \phi(Y) d P$ for all concave $\phi: \mathbb{R} \rightarrow \mathbb{R}([\mathrm{CR}]$ and $[\mathrm{Cn}]$ denote this relation by $Y^{\prime} \prec Y$ and call it majorization), and $F_{f}^{-1}(t)=$ $\inf \left\{z \in \mathbb{R}: F_{f}(z) \geq t\right\}$ for all $t \in[0,1]$.

${ }^{20}$ Let $Y^{\prime} \succeq_{c} Y$. If $\Gamma(Y)=\infty$, then $\Gamma\left(Y^{\prime}\right) \leq \Gamma(Y)$. Else $Y \geqslant 0$ and $\mathrm{E}[Y]=1$, then also $Y^{\prime} \geqslant 0$ and $\mathrm{E}\left[Y^{\prime}\right]=1$ (see $[\mathrm{CR}, \mathrm{p} .62]$ ). The function $\phi(t)=-\frac{1}{2 \theta}\left(t^{2}-1\right)$ is concave, whence $\mathrm{E}\left[\phi\left(Y^{\prime}\right)\right] \geq \mathrm{E}[\phi(Y)]$, and $\Gamma(Y)=-\mathrm{E}[\phi(Y)] \geq-\mathrm{E}\left[\phi\left(Y^{\prime}\right)\right]=\Gamma\left(Y^{\prime}\right)$.
} 
Moreover, for all $Y \in \mathcal{L}^{2}(P)$ there exists $Y^{\prime} \in \mathcal{L}^{2}(P)$ with $Y^{\prime} \succeq_{c} Y$ such that $\int_{0}^{1} F_{f}^{-1}(1-u) F_{Y}^{-1}(u) d u=\int f Y^{\prime} d P$. Since $\Gamma\left(Y^{\prime}\right) \leq \Gamma(Y)$, then

$\int_{0}^{1} F_{f}^{-1}(1-u) F_{Y}^{-1}(u) d u+\Gamma(Y)=\int f Y^{\prime} d P+\Gamma(Y) \geq \int f Y^{\prime} d P+\Gamma\left(Y^{\prime}\right)$,

hence

$$
V_{\theta}(f)=\inf _{Y \in \mathcal{L}^{2}(P)}\left\{\int_{0}^{1} F_{f}^{-1}(1-u) F_{Y}^{-1}(u) d u+\Gamma(Y)\right\} .
$$

If $f \succeq_{c} g$, an inequality of Hardy (see, e.g., [CR, p. 57-58]) delivers

$$
\int_{0}^{1} F_{f}^{-1}(1-u) F_{Y}^{-1}(u) d u \geq \int_{0}^{1} F_{g}^{-1}(1-u) F_{Y}^{-1}(u) d u \quad \forall Y \in \mathcal{L}^{2}(P),
$$

and $V_{\theta}(f) \geq V_{\theta}(g)$. Let $f \succeq_{S S D} g$, Theorem 1.1 of [Cn] guarantees that $f-h \succeq_{c} g$ for some $h \in \mathcal{L}_{+}^{2}(P)$. Since $V_{\theta}$ is monotone, then $V_{\theta}(f) \geq$ $V_{\theta}(f-h) \geq V_{\theta}(g)$.

Proof of Theorem 6. The maximization problem is

$$
\sup _{\alpha \in \mathbb{R}} V_{\theta}\left(W_{\alpha}\right)
$$

where $W_{\alpha}=R+\alpha \cdot(X-\overrightarrow{1} R)$ (remember that $\alpha \in \mathbb{R}^{n}, X \in \mathcal{L}^{2}(P)^{n}$, and $\overrightarrow{1}$ is a vector of $1 \mathrm{~s})$. From Theorem 19 we know that $V_{\theta}$ is Gateaux differentiable and

$$
\nabla V_{\theta}\left(W_{\alpha}\right)=\left(\frac{1}{P\left(W_{\alpha} \leq \kappa_{\alpha}\right)}-\theta\left(W_{\alpha}-\mathrm{E}\left[W_{\alpha} \mid W_{\alpha} \leq \kappa_{\alpha}\right]\right)\right) 1_{\left\{W_{\alpha} \leq \kappa_{\alpha}\right\}}
$$

where $\kappa_{\alpha}$ solves:

$$
P\left(W_{\alpha} \leq \kappa_{\alpha}\right)\left(\kappa_{\alpha}-\mathrm{E}^{P}\left[W_{\alpha} \mid W_{\alpha} \leq \kappa_{\alpha}\right]\right)=\frac{1}{\theta} .
$$

Since for all $i=1, \ldots, n$

$$
\frac{\partial V_{\theta}\left(W_{\alpha}\right)}{\partial \alpha_{i}}=\mathrm{E}\left[\nabla V_{\theta}\left(W_{\alpha}\right)\left(X_{i}-R\right)\right]
$$

the first order conditions for an optimum are:

$$
\mathrm{E}\left[X \nabla V_{\theta}\left(W_{\alpha}\right)\right]=\overrightarrow{1} R
$$


Substituting $\nabla V_{\theta}\left(W_{\alpha}\right)$ :

$\mathrm{E}\left[\frac{1_{\left\{W_{\alpha} \leq \kappa_{\alpha}\right\}}}{P\left(W_{\alpha} \leq \kappa_{\alpha}\right)} X-\theta\left((\alpha \cdot X) 1_{\left\{W_{\alpha} \leq \kappa_{\alpha}\right\}} X-\mathrm{E}\left[\alpha \cdot X \mid W_{\alpha} \leq \kappa_{\alpha}\right] 1_{\left\{W_{\alpha} \leq \kappa_{\alpha}\right\}} X\right)\right]=\overrightarrow{1} R$ set $A=\left\{W_{\alpha} \leq \kappa_{\alpha}\right\}$ to obtain

$$
\mathrm{E}[X \mid A]-\theta P(A)(\mathrm{E}[(\alpha \cdot X) X \mid A]-\mathrm{E}[X \mid A] \mathrm{E}[\alpha \cdot X \mid A])=\overrightarrow{1} R
$$

the observation that $\mathrm{E}[(\alpha \cdot X) X \mid A]-\mathrm{E}[X \mid A] \mathrm{E}[\alpha \cdot X \mid A]=\operatorname{Var}[X \mid A] \alpha$ yields:

$$
\mathrm{E}\left[X-\overrightarrow{1} R \mid W_{\alpha} \leq \kappa_{\alpha}\right]=\theta P\left(W_{\alpha} \leq \kappa_{\alpha}\right) \operatorname{Var}\left[X \mid W_{\alpha} \leq \kappa_{\alpha}\right] \alpha .
$$

These are the first $n$ equations. The $(n+1)$-th is the equation which determines $\kappa_{\alpha}$, that is (33) or (see Lemma 13)

$$
\mathrm{E}\left[\left(W_{\alpha}-\kappa_{\alpha}\right)^{-}\right]=\frac{1}{\theta}
$$

Concavity of $V_{\theta}$ guarantees the sufficiency of first order conditions.

Proof of Proposition 7. Set $\alpha^{*}=\alpha_{m m v}^{*}$. The maximization problem it solves is

$$
\max _{\alpha \in \mathbb{R}} \min _{Y \in \mathbb{Y}}\left(\mathrm{E}[(R+\alpha(X-R)) Y]+\frac{1}{2 \theta} \mathrm{E}\left[Y^{2}\right]-\frac{1}{2 \theta}\right)
$$

where $\mathbb{Y}=\left\{Y \in \mathbb{R}_{+}^{S}: \mathrm{E}[Y]=1\right\}$. Clearly, $\mathbb{Y}$ is convex and compact, and $\left(\alpha^{*}, Y^{*}\right)$ is a solution of (35) if and only if it is a solution of

$$
\max _{\alpha \in \mathbb{R}} \min _{Y \in \mathbb{Y}} G(\alpha, Y)
$$

where $G(\alpha, Y)=\mathrm{E}[(R+\alpha(X-R)) Y]+\frac{1}{2 \theta} \mathrm{E}\left[Y^{2}\right]$. Moreover, notice that $G: \mathbb{R} \times \mathbb{Y} \rightarrow \mathbb{R}$ is continuous, it is affine in $\alpha$ (for each fixed $Y$ ) and strictly convex in $Y$ (for each fixed $\alpha$ ). Set $v=\max _{\alpha \in \mathbb{R}} \min _{Y \in \mathbb{Y}} G(\alpha, Y$ ), by (a version of) the Min-Max Theorem (e.g. [Au, p. 134]) there exists $\bar{Y} \in \mathbb{Y}$ such that

$$
v=\sup _{\alpha \in \mathbb{R}} G(\alpha, \bar{Y}) .
$$

Moreover,

$$
G\left(\alpha^{*}, \bar{Y}\right) \geq \min _{Y \in \mathbb{Y}} G\left(\alpha^{*}, Y\right)=G\left(\alpha^{*}, Y^{*}\right)=v=\sup _{\alpha \in \mathbb{R}} G(\alpha, \bar{Y}) \geq G\left(\alpha^{*}, \bar{Y}\right),
$$


therefore, $G\left(\alpha^{*}, \bar{Y}\right)=\min _{Y \in \mathbb{Y}} G\left(\alpha^{*}, Y\right)$, strict convexity implies $\bar{Y}=Y^{*}$. In turn, this yields $\sup _{\alpha \in \mathbb{R}} G\left(\alpha, Y^{*}\right)=v \neq \infty$ and it cannot be

$$
\sup _{\alpha \in \mathbb{R}}\left(R+\alpha \mathrm{E}\left[(X-R) Y^{*}\right]+\frac{1}{2 \theta} \mathrm{E}\left[\left(Y^{*}\right)^{2}\right]\right)=\infty,
$$

therefore

$$
\mathrm{E}\left[Y^{*}(X-R)\right]=0 .
$$

$Y^{*}$ is the solution of problem (25) in the proof of Theorem 19 with $f=$ $R+\alpha^{*}(X-R)=W_{\alpha^{*}}$. Therefore, there exist $\lambda^{*} \in \mathbb{R}$ and $\mu \in \mathcal{L}^{2}(P)$ $\left(=\mathbb{R}^{S}\right)$ such that $Y^{*}$ satisfies the following conditions:

$$
\begin{aligned}
& R+\alpha^{*}(X-R)+\frac{1}{\theta} Y^{*}-\mu-\lambda^{*}=0, \\
& \mathrm{E}\left[Y^{*}\right]=1 \\
& Y^{*} \geqslant 0, \mu \geqslant 0, \mu Y^{*}=0
\end{aligned}
$$

Taking the expectation of both sides of (37) we obtain:

$$
\left(1-\alpha^{*}\right) R+\alpha^{*} \mathrm{E}[X]+\frac{1}{\theta} \mathrm{E}\left[Y^{*}\right]-\mathrm{E}[\mu]-\lambda^{*}=0
$$

and, subtracting (40) from (37):

$$
\alpha^{*}(X-\mathrm{E}[X])+\frac{1}{\theta}\left(Y^{*}-\mathrm{E}\left[Y^{*}\right]\right)-(\mu-\mathrm{E}[\mu])=0 .
$$

Rearranging and using (38):

$$
Y^{*}=1-\theta \alpha^{*}(X-\mathrm{E}[X])+\theta(\mu-\mathrm{E}[\mu]) .
$$

Multiply both sides by $\mu$, take expectations and use (39) to get:

$$
\mathrm{E}[\mu]-\theta \alpha^{*} \operatorname{Cov}[\mu, X]+\theta \operatorname{Var}[\mu]=0
$$

and, rearranging terms:

$$
\theta \alpha^{*} \operatorname{Cov}[\mu, X]=\mathrm{E}[\mu]+\theta \operatorname{Var}[\mu] .
$$

Since $\mu \geqslant 0$, then $\mathrm{E}[\mu] \geq 0$ thus:

$$
\begin{aligned}
& \alpha^{*}=0 \Rightarrow \mu=0 \Rightarrow \operatorname{Cov}[\mu, X]=0 \\
& \alpha^{*}>0 \Rightarrow \operatorname{Cov}[\mu, X] \geq 0 \\
& \alpha^{*}<0 \Rightarrow \operatorname{Cov}[\mu, X] \leq 0
\end{aligned}
$$


Now, plugging (41) into (36) we obtain:

$$
\mathrm{E}\left[\left(1-\theta \alpha^{*}(X-\mathrm{E}[X])+\theta(\mu-\mathrm{E}[\mu])\right) X\right]=R
$$

or:

$$
\mathrm{E}[X]-\theta \alpha^{*} \operatorname{Var}[X]+\theta \operatorname{Cov}[\mu, X]=R
$$

which becomes:

$$
\alpha^{*}=\frac{1}{\theta} \frac{\mathrm{E}[X-R]}{\operatorname{Var}[X]}+\frac{\operatorname{Cov}[\mu, X]}{\operatorname{Var}[X]}
$$

Recalling that:

$$
\alpha_{m v}^{*}=\frac{1}{\theta} \frac{\mathrm{E}[X-R]}{\operatorname{Var}[X]}
$$

we obtain:

$$
\alpha^{*}=\alpha_{m v}^{*}+\frac{\operatorname{Cov}[\mu, X]}{\operatorname{Var}[X]}
$$

Using (43) - (45), it is now obvious that:

$$
\begin{aligned}
& \alpha^{*}=0 \Rightarrow \alpha^{*}=\alpha_{m v}^{*}=0 \\
& \alpha^{*}>0 \Rightarrow \alpha^{*} \geq \alpha_{m v}^{*} \\
& \alpha^{*}<0 \Rightarrow \alpha^{*} \leq \alpha_{m v}^{*}
\end{aligned}
$$

From the proof of Theorem 19 - Eq. $(31)$ - we know that $\lambda^{*}=g_{W_{\alpha^{*}}}^{-1}\left(\frac{1}{\theta}\right)=\kappa^{*}$. Furthermore, if $P\left(W_{\alpha^{*}}>\kappa^{*}\right)>0$, since $S$ is finite, there exists $s$ such that

$$
R+\alpha^{*}(X(s)-R)=W_{\alpha^{*}}(s)>\kappa^{*}=\lambda^{*},
$$

that is $R+\alpha^{*}(X(s)-R)-\lambda^{*}>0$. Since $Y^{*}(s) \geq 0$, by (37), we have

$$
\mu(s)=R+\alpha^{*}(X(s)-R)-\lambda^{*}+\frac{1}{\theta} Y^{*}>0,
$$

and $\mathrm{E}[\mu]>0$. Thus, in this case (42) implies $\alpha^{*} \operatorname{Cov}[\mu, X]>0$ and the inequalities in (48) and (49) become strict.

Finally, we want to show that $\alpha^{*} \alpha_{m v}^{*} \geq 0$. By contradiction, suppose $\alpha^{*} \alpha_{m v}^{*}<0$. Then, either $\alpha^{*}>0$ and $\alpha_{m v}^{*}<0$ or $\alpha^{*}<0$ and $\alpha_{m v}^{*}>0$. Suppose $\alpha^{*}>0$ and $\alpha_{m v}^{*}<0$, since

$$
\alpha_{m v}^{*}=\frac{1}{\theta} \frac{\mathrm{E}[X-R]}{\operatorname{Var}[X]}
$$

it must be $\mathrm{E}[X-R]<0$ and

$$
\alpha^{*} \mathrm{E}[X-R]<0 .
$$


Clearly, if $\alpha^{*}<0$ and $\alpha_{m v}^{*}>0,(50)$ still holds. Remember that $\left(\alpha^{*}, Y^{*}\right)$ is a saddle point for

$$
G(\alpha, Y)=\mathrm{E}[(R+\alpha(X-R)) Y]+\frac{1}{2 \theta} \mathrm{E}\left[Y^{2}\right]
$$

and so:

$$
G\left(\alpha^{*}, Y^{*}\right) \leq G\left(\alpha^{*}, 1_{S}\right)=R+\alpha^{*} \mathrm{E}[X-R]<R
$$

where the last inequality follows from (50). But,

$$
\begin{aligned}
\min _{Y \in \mathbb{Y}} G(0, Y) & =R+\min _{Y \in \mathbb{Y}} \frac{1}{2 \theta} \mathrm{E}\left[Y^{2}\right] \geq R \\
& >G\left(\alpha^{*}, Y^{*}\right)=\max _{\alpha \in \mathbb{R}} \min _{Y \in \mathbb{Y}} G(\alpha, Y) \geq \min _{Y \in \mathbb{Y}} G(0, Y),
\end{aligned}
$$

which is impossible.

Proof of Proposition 8. Assume

$$
\left\{\begin{array}{l}
\theta P\left(W_{\alpha^{\theta}} \leq \kappa^{\theta}\right) \operatorname{Var}\left[X \mid W_{\alpha^{\theta}} \leq \kappa^{\theta}\right] \alpha^{\theta}=\mathrm{E}\left[X-\overrightarrow{1} R \mid W_{\alpha^{\theta}} \leq \kappa^{\theta}\right] \\
\mathrm{E}\left[\left(W_{\alpha^{\theta}}-\kappa^{\theta}\right)^{-}\right]=\frac{1}{\theta}
\end{array}\right.
$$

Set

$$
\alpha^{\gamma}=\frac{\theta}{\gamma} \alpha^{\theta} \text { and } \kappa^{\gamma}=\frac{\theta}{\gamma}\left(\kappa^{\theta}-R\right)+R
$$

Then,

$$
\begin{aligned}
W_{\alpha^{\theta}} & =R+\alpha^{\theta} \cdot(X-\overrightarrow{1} R)=R+\frac{\gamma}{\theta} \alpha^{\gamma} \cdot X-\frac{\gamma}{\theta} \alpha^{\gamma} \cdot \overrightarrow{1} R \\
& =R-\frac{\gamma}{\theta} R+\frac{\gamma}{\theta} R+\frac{\gamma}{\theta} \alpha^{\gamma} \cdot X-\frac{\gamma}{\theta} \alpha^{\gamma} \cdot \overrightarrow{1} R \\
& =\frac{\gamma}{\theta} W_{\alpha^{\gamma}}+R\left(1-\frac{\gamma}{\theta}\right)
\end{aligned}
$$

and so

$$
W_{\alpha^{\theta}}=\frac{\gamma}{\theta} W_{\alpha^{\gamma}}+R\left(1-\frac{\gamma}{\theta}\right) \text { and } \kappa^{\theta}=\frac{\gamma}{\theta} \kappa^{\gamma}+R\left(1-\frac{\gamma}{\theta}\right)
$$

Equation (51) becomes

$$
\left\{\begin{array}{l}
\theta P\left(W_{\alpha^{\gamma}} \leq \kappa^{\gamma}\right) \operatorname{Var}\left[X \mid W_{\alpha^{\gamma}} \leq \kappa^{\gamma}\right] \frac{\gamma}{\theta} \alpha^{\gamma}=\mathrm{E}\left[X-\overrightarrow{1} R \mid W_{\alpha^{\gamma}} \leq \kappa^{\gamma}\right] \\
\mathrm{E}^{P}\left[\left(\frac{\gamma}{\theta}\left(W_{\alpha^{\gamma}}-\kappa^{\gamma}\right)\right)^{-}\right]=\frac{1}{\theta} .
\end{array}\right.
$$


that is

$$
\left\{\begin{array}{l}
\gamma P\left(W_{\alpha \gamma} \leq \kappa^{\gamma}\right) \operatorname{Var}\left[X \mid W_{\alpha^{\gamma}} \leq \kappa^{\gamma}\right] \alpha^{\gamma}=\mathrm{E}\left[X-\overrightarrow{1} R \mid W_{\alpha^{\gamma}} \leq \kappa^{\gamma}\right] \\
\mathrm{E}\left[\left(W_{\alpha \gamma}-\kappa^{\gamma}\right)^{-}\right]=\frac{1}{\gamma} .
\end{array}\right.
$$

so that $\left(\alpha^{\gamma}, \kappa^{\gamma}\right)$ solves the portfolio selection problem for an investor with uncertainty aversion $\gamma$.

Proof of Lemma 9. Consider the portfolio selection problem (6) with $\theta=1$. Next we show that

$$
\max _{\alpha \in \mathbb{R}^{n}} V_{1}\left(W_{\alpha}\right)=\max _{\zeta \in \mathbb{R}} V_{1}\left(\zeta X_{m}+(1-\zeta) R\right)
$$

and $m \in \arg \max _{\zeta \in \mathbb{R}} V_{1}\left(\zeta X_{m}+(1-\zeta) R\right)$.

First observe that, for all $\zeta \in \mathbb{R}$,

$$
\zeta X_{m}+(1-\zeta) R=\zeta\left(\alpha^{m} \cdot X\right)+\left(1-\zeta \alpha^{m} \cdot \overrightarrow{1}\right) R=W_{\zeta \alpha^{m}}
$$

hence $\max _{\zeta \in \mathbb{R}} V_{1}\left(\zeta X_{m}+(1-\zeta) R\right) \leq \max _{\alpha \in \mathbb{R}^{n}} V_{1}\left(W_{\alpha}\right)$. Conversely, if $\alpha^{1} \in$ $\arg \max _{\alpha \in \mathbb{R}^{n}} V_{1}\left(W_{\alpha}\right)$, then $m=\alpha^{1} \cdot \overrightarrow{1}$ and $\alpha^{m}=\alpha^{1} /\left(\alpha^{1} \cdot \overrightarrow{1}\right)$ or $\alpha^{1}=m \alpha^{m}$. Hence

$$
\begin{aligned}
\max _{\alpha \in \mathbb{R}^{n}} V_{1}\left(W_{\alpha}\right) & =V_{1}\left(W_{\alpha^{1}}\right)=V_{1}\left(W_{m \alpha^{m}}\right)=V_{1}\left(m \alpha^{m} \cdot X+\left(1-m \alpha^{m} \cdot \overrightarrow{1}\right) R\right) \\
& =V_{1}\left(m X_{m}+(1-m) R\right) \leq \max _{\zeta \in \mathbb{R}} V_{1}\left(\zeta X_{m}+(1-\zeta) R\right) \\
& \leq \max _{\alpha \in \mathbb{R}^{n}} V_{1}\left(W_{\alpha}\right)
\end{aligned}
$$

as wanted. Now, applying Theorem 6 with $X_{m}$ instead of $X, m$ must satisfy the following conditions:

$\left\{\begin{array}{l}P\left(m X_{m}+(1-m) R \leq \nu\right) \operatorname{Var}\left[X_{m} \mid m X_{m}+(1-m) R \leq \nu\right] m=\mathrm{E}\left[X_{m}-R \mid m X_{m}+(1-m) R \leq \nu\right], \\ \mathrm{E}\left[\left(m X_{m}+(1-m) R-\nu\right)^{-}\right]=1 .\end{array}\right.$

Moreover, since $X_{m}$ is the (optimal) final wealth of an agent with initial wealth 1 and uncertainty aversion coefficient $m$, again by Theorem 6 applied to such an agent it must be the case that

$$
\mathrm{E}\left[\left(X_{m}-\kappa^{m}\right)^{-}\right]=1 / m
$$

But the second equation of previous system is equivalent to

$$
\mathrm{E}\left[\left(X_{m}-\left(\frac{\nu}{m}-\frac{(1-m)}{m} R\right)\right)^{-}\right]=\frac{1}{m}
$$


and since $g_{X_{m}}$ is strictly increasing (see Lemma 14), then

$$
\frac{\nu}{m}-\frac{(1-m)}{m} R=\kappa^{m}
$$

By (56), $\left\{m X_{m}+(1-m) R \leq \nu\right\}=\left\{X_{m} \leq \frac{\nu}{m}-\frac{(1-m)}{m} R\right\}=\left\{X_{m} \leq \kappa^{m}\right\}$ and (55) is equivalent to (54), thus (53) amounts to

$$
\left\{\begin{array}{l}
P\left(X_{m} \leq \kappa^{m}\right) \operatorname{Var}\left[X_{m} \mid X_{m} \leq \kappa^{m}\right] m=\mathrm{E}\left[X_{m}-R \mid X_{m} \leq \kappa^{m}\right] \\
\mathrm{E}\left[\left(X_{m}-\kappa^{m}\right)^{-}\right]=1 / m
\end{array}\right.
$$

as wanted.

Proof of Lemma 10. (i) First observe that by Remark 20 and Eq. (52) in the proof of Proposition 8, for all $\theta>0$

$$
\begin{aligned}
\nabla V_{\theta}\left(W_{\alpha^{\theta}}\right) & =\theta\left(\kappa^{\theta}-W_{\alpha^{\theta}}\right) 1_{\left\{W_{\left.\alpha^{\theta} \leq \kappa^{\theta}\right\}}\right.}=\theta\left(\frac{m}{\theta} \kappa^{m}-\frac{m}{\theta} W_{\alpha^{m}}\right) 1_{\left\{W_{\left.\alpha^{m} \leq \kappa^{m}\right\}}\right.} \\
& =m\left(\kappa^{m}-X_{m}\right) 1_{\left\{X_{m} \leq \kappa^{m}\right\}}=m\left(X_{m}-\kappa^{m}\right)^{-}=\nabla V_{m}\left(X_{m}\right) .
\end{aligned}
$$

(ii) The first order conditions for an optimum are $\mathrm{E}\left[X \nabla V_{m}\left(W_{\alpha^{m}}\right)\right]=\overrightarrow{1} R$ (see Eq. 34).

(iii) Descends from Remark 18.

\section{References}

[Au] Aubin, J-P., Optima and equilibria, Springer-Verlag, Berlin, 1998.

[Bi] Bigelow, J. P., Consistency of mean-variance analysis and expected utility analysis: a complete characterisation, Economic Letters, 43, 187-192, 1993.

[Br] Britten-Jones, M., The sampling error in estimates of mean-variance efficient portfolio weights, Journal of Finance, 45, 655-671, 1999.

[BKM] Bodie, Z., A. Kane, and A. J. Marcus, Investments, McGraw Hill, Boston, 2002.

[CR] Chong, K. M. and N. M. Rice, Equimeasurable rearrangements of functions, Queens Papers in Pure and Applied Mathematics, 28, 1971. 
[Cn] Chong, K. M., Doubly stochastic operators and rearrangement theorems, Journal of Mathematical Analysis and Applications, 56, 309316, 1976.

[Ch] Choquet, G., Lectures on Analysis (vol. 2), Benjamin, New York, 1969 .

[Da] Dana, R-A., A representation result for concave Schur concave functions, Mathematical Finance, 15, 613-634, 2005.

[DI] Dybvig, P. H. and J. E. Ingersoll, Mean-Variance theory in complete markets, The Journal of Business, 55, 233-251, 1982.

[DR] Dybvig, P. H. and S. A. Ross, Arbitrage, in New Palgrave: A Dictionary of Economics, J. Eatwell, M. Milgate, and P. Newman (eds.), MacMillan, London, 1987.

[FK] Filipovic, D. and M. Kupper, Equilibrium Prices for Monetary Utility Functions, mimeo, 2006.

[FK-2] Filipovic, D. and M. Kupper, Monotone and cash-invariant convex functions and hulls, mimeo, 2006.

[GS] Gilboa, I. and D. Schmeidler, Maxmin expected utility with nonunique prior, Journal of Mathematical Economics, 18, 141-153, 1989.

[GRS] Gibbons, M. R., S. A. Ross and J. Shanken, A test of the efficiency of a given portfolio, Econometrica, 57, 1121-1152, 1989.

[HS] Hansen, L. and T. Sargent, Robust control and model uncertainty, American Economic Review, 91, 60-66, 2001.

[JM] Jarrow, R. A. and D. B. Madan, Is Mean-Variance analysis vacuous: Or was Beta still born?, European Finance Review, 1, 15-30, 1997.

[KS] Kandel, S. and R. Stambaugh, A mean-variance framework for tests of asset pricing models, Review of Financial Studies, 2, 125-156, 1987.

[KC] Kupper, M. and P. Cheridito, Time-consistency of indifference prices and monetary utility functions, mimeo, 2006.

[LV] Liese, F. and I. Vajda, Convex Statistical Distances, Teubner, Leipzig, 1987. 
[MMR] Maccheroni, F., M. Marinacci and A. Rustichini, Ambiguity aversion, robustness, and the variational representation of preferences, Econometrica, 74, 1447-1498, 2006.

[MMR-2] Maccheroni, F., M. Marinacci, and A. Rustichini, Dynamic variational preferences, Journal of Economic Theory, 128, 4-44, 2006.

[MR] MacKinlay, A. C. and M. P. Richardson, Using generalized method of moments to test mean-variance efficiency, Journal of Finance, 46, 511-527, 1991.

[Ph] Phelps, R. R., Convex functions, monotone operators and differentiability, Springer-Verlag, New York, 1992.

[Ma] Markowitz, H. M., Portfolio selection, Journal of Finance, 7, 77-91, 1952.

[R] Ross S. A., Neoclassical Finance, Princeton University Press, Princeton, 2005.

[Sh] Sharpe, W. F., Capital asset prices: a theory of market equilibrium under conditions of risk, Journal of Finance, 19, 425-442, 1964.

[Sh-2] Sharpe, W. F., Capital asset prices with and without negative holdings, Journal of Finance, 46, 489-509,1991.

[To] Tobin, J., Liquidity preference as behavior towards risk, Review of Economic Studies, 25, 65-86, 1958. 